\title{
COMMUTATOR THEORY FOR RELATIVELY MODULAR QUASIVARIETIES
}

\author{
KEITH KEARNES AND RALPH MCKENZIE
}

\begin{abstract}
We develop a commutator theory for relatively modular quasivarieties that extends the theory for modular varieties. We characterize relatively modular quasivarieties, prove that they have an almost-equational axiomatization and we investigate the lattice of subquasivarieties. We derive the result that every finitely generated, relatively modular quasivariety of semigroups is finitely based.
\end{abstract}

\section{INTRODUCTION}

A quasivariety is a class of similar algebras closed under the formation of subalgebras, products, and ultraproducts; equivalently, a class defined by a set of quasi-equations. For a quasivariety $\mathscr{K}$ and any algebra $\mathbf{A}$ similar to the algebras in $\mathscr{K}$, we define Con $\mathbf{A}$ to be the lattice of all congruence relations of $\mathbf{A}$ and $\operatorname{Con}_{\mathscr{K}} \mathbf{A}$ to be the lattice consisting of all $\theta \in \mathbf{C o n} \mathbf{A}$ such that $\mathbf{A} / \theta \in \mathscr{K}$. The meet in $\operatorname{Con}_{\mathscr{K}} \mathbf{A}$ is the same as in $\operatorname{Con} \mathbf{A}$, but the join is different. The members of $\operatorname{Con}_{\mathscr{K}} \mathbf{A}$ will be called $\mathscr{K}$-congruences of $\mathbf{A}$. The least $\mathscr{K}$-congruence of $\mathbf{A}$ containing a set $T \subseteq A^{2}$ will be denoted $\mathrm{Cg}_{\mathscr{K}}(T)$; then $\mathrm{Cg}_{\mathscr{H}}$ is an algebraic closure operator on $A^{2}$ and $\operatorname{Con}_{\mathscr{H}} \mathbf{A}$ is the lattice of closed sets for this operator.

A quasivariety $\mathscr{K}$ will be said to be relatively modular (relatively distributive) iff for every $\mathbf{A} \in \mathscr{K}$ the lattice $\operatorname{Con}_{\mathscr{K}} \mathbf{A}$ is a modular (respectively, distributive) lattice. Our route to a commutator theory for relatively modular quasivarieties will parallel the way taken in [FM] to develop the commutator theory for congruence-modular varieties. For a relatively modular quasivariety that happens to be a variety, our commutator will be the same as the old one. Our motivation to seek a commutator theory for quasivarieties sprang from two recent results. In [M], R. McKenzie proved that a variety of finite type generated by a finite algebra is finitely axiomatizable provided the variety is congruencemodular and residually small. In [P], D. Pigozzi proved that every relatively distributive quasivariety generated as a quasivariety by a finite set of finite similar algebras of finite type is finitely axiomatizable. These two theorems have a natural join, every relatively modular quasivariety of finite type generated by a finite set of finite algebras is finitely axiomatizable. The italicized statement is

Received by the editors July 5, 1989 and, in revised form, June 5, 1990. 1980 Mathematics Subject Classification (1985 Revision). Primary 08B10, $08 \mathrm{C} 15$.

Research of the second author supported by National Science Foundation grant DMS 8302255. 
still a conjecture; but we believe that the commutator theory developed in this paper will lead to a proof of it.

The first theorem gives a rather surprising result that is quite useful.

Theorem 1.1. Suppose that the quasivariety $\mathscr{K}$ is relatively modular. For every $\mathbf{A}$ in $\mathscr{K}$, the operator $\mathrm{Cg}_{\mathscr{K}}$ restricted to $\mathbf{C o n} \mathbf{A}$ is a lattice homomorphism of Con A onto Con $_{\mathscr{K}} \mathbf{A}$.

Proof. Assume that $\mathscr{K}$ is relatively modular and that $\mathbf{A} \in \mathscr{K}$. For any quasivariety, $\mathrm{Cg}_{\mathscr{K}}$ is a complete join homomorphism. We must prove that for $\alpha, \beta \in \mathrm{Con} \mathbf{A}$, we have $\mathrm{Cg}_{\mathscr{H}}(\alpha \cdot \beta)=\mathrm{Cg}_{\mathscr{H}}(\alpha) \cdot \mathrm{Cg}_{\mathscr{H}}(\beta)$. To do so, we consider the algebra $\mathbf{C}$ consisting of all ordered triples $(x, y, z) \in A^{3}$ such that $(x, y) \in \alpha$ and $(y, z) \in \beta$ (a subalgebra of $\mathbf{A}^{3}$ ). Since $\mathbf{C} \in \mathscr{K}, \mathbf{C o n}_{\mathscr{K}} \mathbf{C}$ is modular. We consider three members of this modular lattice, namely the kernels $\eta_{0}, \eta_{1}, \eta_{2}$ of the three coordinate projections $p_{0}, p_{1}, p_{2}$ of $\mathbf{C}$ onto A.

For congruences $\psi, \theta$ of an algebra, we shall write $\theta^{\prime}$ in place of $\operatorname{Cg}_{\mathscr{K}}(\theta)$ and $\theta+{ }^{\prime} \psi$ in place of $\operatorname{Cg}_{\mathscr{H}}(\theta+\psi)$. For $i \in\{0,1,2\}$ and for $\theta \in \operatorname{Con} \mathbf{A}$, we shall write $\theta_{i}$ for $p_{i}^{-1}(\theta)$, a congruence of $\mathbf{C}$. Note that $\theta_{i} \in \operatorname{Con}_{\mathscr{H}} \mathbf{C}$ iff $\theta \in$ $\operatorname{Con}_{\mathscr{K}} \mathbf{A}$, since $p_{i}$ maps $\mathbf{C}$ homomorphically onto $\mathbf{A}$. From this observation, it easily follows that $\left(\theta^{\prime}\right)_{i}=\left(\theta_{i}\right)^{\prime}$.

Our first calculations, involving congruences of $\mathbf{C}$ and their $\mathscr{K}$-extensions, will show that $\left(\alpha \beta^{\prime}\right)^{\prime}=\alpha^{\prime} \beta^{\prime}$. Note that $\eta_{0} \leq \alpha_{1}$, and that

$$
\eta_{0}+\eta_{1}=\alpha_{0}=\alpha_{1}, \quad \eta_{0}{ }^{\prime} \eta_{1}=\alpha_{0}^{\prime}=\alpha_{1}^{\prime}, \quad \eta_{1}{ }^{\prime}{ }^{\prime} \eta_{2}=\beta_{1}^{\prime} .
$$

In the calculation that follows, the third equality is a consequence of the modularity of $\operatorname{Con}_{\mathscr{K}} \mathbf{C}$, and the final equality becomes obvious by working with the elements.

$$
\begin{aligned}
\left(\alpha^{\prime} \beta^{\prime}\right)_{1} & =\left(\alpha_{1}\right)^{\prime}\left(\beta_{1}\right)^{\prime}=\left(\eta_{0}+^{\prime} \eta_{1}\right)\left(\eta_{1}+^{\prime} \eta_{2}\right) \\
& =\eta_{1}+^{\prime} \eta_{0}\left(\eta_{1}+^{\prime} \eta_{2}\right)=\eta_{1}+^{\prime}\left(\eta_{0} \cdot \alpha_{1} \cdot \beta_{1}^{\prime}\right) \\
& =\eta_{1}{ }^{\prime} \eta_{0} \cdot\left(\alpha \beta^{\prime}\right)_{1}=\left(\alpha \beta^{\prime}\right)_{1}^{\prime} .
\end{aligned}
$$

Thus $\left(\alpha \beta^{\prime}\right)^{\prime}=\alpha^{\prime} \beta^{\prime}$.

The formula we have just proved will hold of course for congruences of $\mathbf{C}$. Substituting $\alpha_{1}$ for $\alpha$ and $\eta_{2}$ for $\beta$, and using that $\eta_{2}^{\prime}=\eta_{2}$, we derive

$$
\left(\alpha_{1} \eta_{2}\right)^{\prime}=\left(\alpha_{1} \eta_{2}^{\prime}\right)^{\prime}=\left(\alpha_{1}\right)^{\prime}\left(\eta_{2}\right)^{\prime}=\left(\eta_{0}+{ }^{\prime} \eta_{1}\right) \eta_{2} \text {. }
$$

Now $\alpha_{1} \eta_{2} \leq(\alpha \beta)_{1}$, hence $(\alpha \beta)_{1}^{\prime} \geq\left(\eta_{0}+^{\prime} \eta_{1}\right) \eta_{2}$. Thus

$$
(\alpha \beta)_{1}^{\prime} \geq\left(\eta_{0}+^{\prime} \eta_{1}\right) \eta_{2}+^{\prime} \eta_{1}=\left(\eta_{0}+^{\prime} \eta_{1}\right)\left(\eta_{2}+^{\prime} \eta_{1}\right)=\left(\alpha^{\prime} \beta^{\prime}\right)_{1} \text {. }
$$

Since it is clear that $\alpha^{\prime} \beta^{\prime} \geq(\alpha \beta)^{\prime}$, we obtain that $\alpha^{\prime} \beta^{\prime}=(\alpha \beta)^{\prime}$ as desired.

The property that the operator $\mathrm{Cg}_{\mathscr{K}}$ is a lattice homomorphism from Con $\mathbf{A}$ onto $\operatorname{Con}_{\mathscr{K}} \mathbf{A}$ for each $\mathbf{A} \in \mathscr{K}$ will be called the extension principle. To indicate that $\mathscr{K}$ satisfies the extension principle, we write $\mathscr{K} \models_{\text {con }}$ $\mathrm{Cg}_{\mathscr{H}}(\alpha \cdot \beta)=\mathrm{Cg}_{\mathscr{H}}(\alpha) \cdot \mathrm{Cg}_{\mathscr{H}}(\beta)$.

Henceforth, when working with a single quasivariety $\mathscr{K}$, we shall use $\alpha^{\prime}$ to denote the $\mathscr{K}$-congruence generated by $\alpha$ (or $\mathscr{K}$-extension of $\alpha$ ). We shall also use $\theta+{ }^{\prime} \psi$ to denote the $\mathscr{K}$-congruence join of $\theta$ and $\psi$. If more than one quasivariety is under discussion, we will revert back to $\mathrm{Cg}_{\mathscr{K}}(\alpha)$ to denote the $\mathscr{K}$-extension of $\alpha$ and use $+_{\mathscr{K}}$ to denote the $\mathscr{K}$-join. 
Theorem 1.2. If $\mathscr{K}$ is a locally finite, relatively modular subquasivariety of a semisimple, congruence-modular variety $\mathscr{V}$, then $\mathscr{K}$ is a variety.

Proof. Assume that $\mathscr{K} \neq \boldsymbol{H}(\mathscr{K})$. Then there is a simple algebra $\mathbf{A} \in \boldsymbol{H}(\mathscr{K})-$ $\mathscr{K}$. Now W. Dziobiak proved in [Dz1] that every subdirectly irreducible algebra $\mathbf{X}$ is embeddable into an ultraproduct of finitely generated subdirectly irreducible algebras belonging to $\boldsymbol{H S}(\mathbf{X})$. This allows us to assume that $\mathbf{A}$ is finitely generated. $\boldsymbol{H}(\mathscr{K})$ is a locally finite variety so $\mathbf{A}$ is finite. Choose a finite algebra $\mathbf{B} \in \mathscr{K}$ of least cardinality that has a congruence $\psi$ with the property that $\mathbf{B} / \psi \cong \mathbf{A}$. Our assumptions insure that $\psi$ is a maximal proper congruence on $\mathbf{B}$ and that $\psi$ is not a $\mathscr{K}$-congruence. Notice that if $\theta$ is any completely meet-irreducible member of $\mathbf{C o n}_{\mathscr{H}} \mathbf{B}$ then, by Theorem 1.1, $\theta$ is at least meet-irreducible in Con B ; since B is finite and lies in a semisimple variety, this means that $\theta$ is a maximal proper congruence. Let $\left\{\theta_{i} \mid i<n+1\right\}$ be an irredundant set of completely meet-irreducible $\mathscr{K}$-congruences that separate the points of $\mathbf{B}$. Let $\tilde{\psi}=\bigwedge_{i<n} \theta_{i} . \quad \tilde{\psi}$ is a $\mathscr{K}$-congruence since it is a meet of $\mathscr{K}$-congruences. By the irredundancy of the $\theta_{i}$ and the modularity of $\mathscr{V}, 0 \prec \tilde{\psi}$. Further, $\tilde{\psi} \nless \psi$ by the minimality of $|B|$. Hence, 0 $=\mathrm{Cg}_{\mathscr{H}}(0)=\mathrm{Cg}_{\mathscr{H}}(\psi \cdot \tilde{\psi}) \neq \mathrm{Cg}_{\mathscr{H}}(\psi) \cdot \mathrm{Cg}_{\mathscr{H}}(\tilde{\psi})=1 \cdot \tilde{\psi}=\tilde{\psi}$. This is a contradiction.

An analogue of Theorem 1.2 for relatively distributive quasivarieties can be found in [Dz2].

Example 1.3. The lattice $\mathbf{M}_{33}^{\prime}$ generates, of course, a semisimple, congruencedistributive variety. We will show that the quasivariety generated by $\mathbf{M}_{33}^{\prime}$ cannot be relatively modular. Let $\mathscr{K}$ be that quasivariety. Now $\mathscr{K}$ is a locally finite subquasivariety of the (semisimple) variety generated by $\mathbf{M}_{33}^{\prime}$. We have that $\mathscr{K}=\boldsymbol{S P P}_{u}\left(\mathbf{M}_{33}^{\prime}\right)=\boldsymbol{S P}\left(\mathbf{M}_{33}^{\prime}\right)$. Since $\mathbf{M}_{33}$ is simple and not embeddable into $\mathbf{M}_{33}^{\prime}$, then $\mathbf{M}_{33} \notin \mathscr{K}$. However, $\mathbf{M}_{33} \in \boldsymbol{H}\left(\mathbf{M}_{33}^{\prime}\right)$, showing that $\mathscr{K}$ is not a variety. By Theorem 1.2, $\mathscr{K}$ cannot be relatively modular. From the proofs of Theorems 1.1 and 1.2, we can conclude that some subdirect power of nine copies of $\mathbf{M}_{33}^{\prime}$ has a nonmodular lattice of $\mathscr{K}$-congruences. In fact, the lattice $\mathbf{L}$ in Figure 2 is a subdirect product of only two copies of $\mathbf{M}_{33}^{\prime}$ and has a nonmodular $\mathscr{K}$-congruence lattice.

In [GL], it is proved that $\boldsymbol{V}\left(\mathbf{M}_{33}\right)$ has $2^{\aleph_{0}}$ subquasivarieties that are distinct from varieties. By Theorem 1.2, none of them are relatively modular.

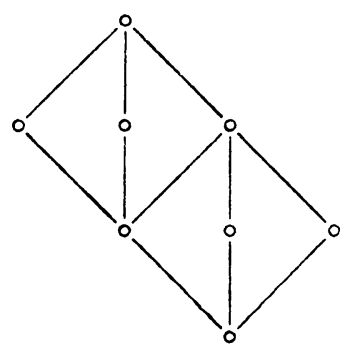

$\mathrm{M}_{33}$

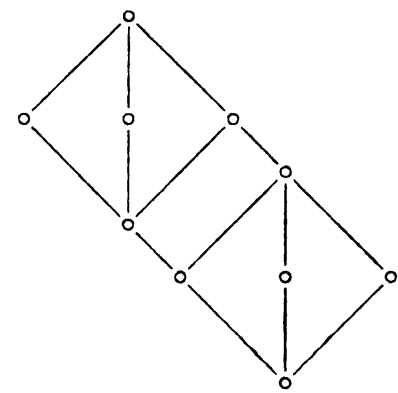

$\mathrm{M}_{33}^{\prime}$ 


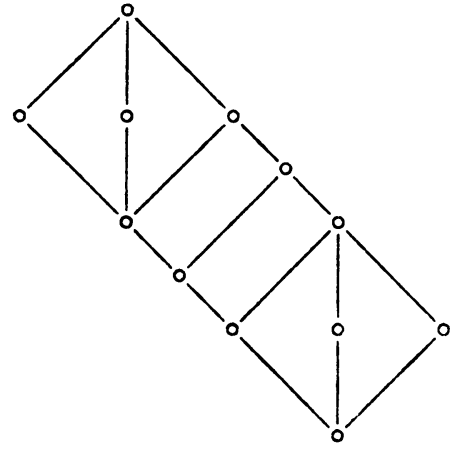

L

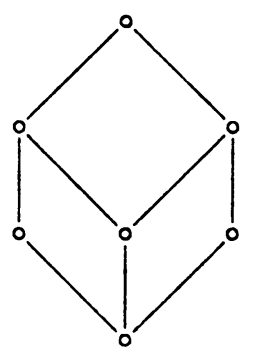

$\operatorname{Con}_{\mathcal{K}} \mathrm{L}$

FIGURE 2

\section{COMMUTATOR THEORY}

Theorem 2.1 (Relative shifting lemma). Let $\mathscr{K}$ be a relatively modular quasivariety.

(1) Suppose that $\mathbf{A} \in \mathscr{K}$, that $\theta_{0}, \theta_{1}, \psi \in \operatorname{Con}_{\mathscr{K}} \mathbf{A}$, and that $a, b, c, d \in$ $A$ satisfy $(a, b),(c, d) \in \theta_{1},(a, d),(b, c) \in \theta_{0}$, and $(b, c) \in \psi$. Then $(a, d) \in \psi+{ }^{\prime} \theta_{0} \theta_{1}$. (This statement is expressed pictorially in Figure 3. In diagrams like this, lines are assumed to be labeled by any label appearing on a parallel line.)

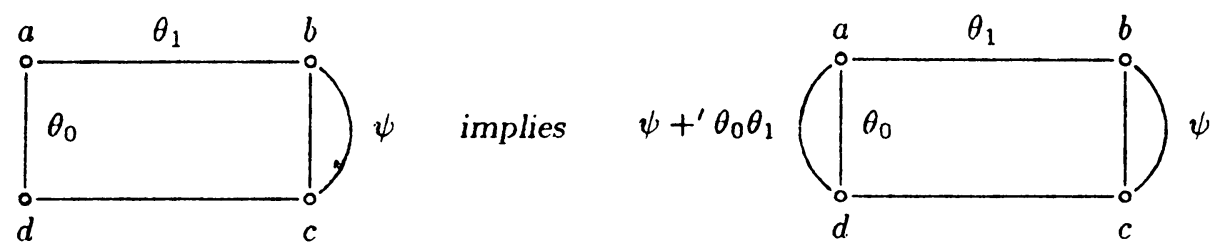

FiguRE 3

(2) There exist a finite set $\Sigma_{s}$ of pairs of terms in the language of $\mathscr{K}$, $\langle p(x, y, z, u), q(x, y, z, u)\rangle$, such that: (i) if $\langle p, q\rangle \in \Sigma_{s}$ then the equations

$$
p(x, y, y, x) \approx q(x, y, y, x) \text { and } p(x, x, y, y) \approx q(x, x, y, y)
$$

are valid in $\mathscr{K}$; and (ii) if $a, b, d$ belong to an algebra $\mathbf{A}$ in $\mathscr{K}$ and

$$
p^{\mathbf{A}}(a, b, b, d)=q^{\mathbf{A}}(a, b, b, d)
$$

for all pairs $(p, q) \in \Sigma_{s}$, then $a=d$.

Proof. The first assertion follows immediately from the modularity of $\operatorname{Con}_{\mathscr{K}} \mathbf{A}$. To prove the second assertion, let $\mathbf{F}$ be the free algebra in the variety generated by $\mathscr{K}$, freely generated by $x, y, z, u$. Then $\mathbf{F}$ is in $\mathscr{K}$, and every congruence of $\mathbf{F}$ generated by some equivalence relation on the four-element set of free generators is a $\mathscr{K}$-congruence (since it is the kernel of an endomorphism of $\mathbf{F})$. Let $\theta_{0}$ be the congruence of $\mathbf{F}$ generated by $\{(x, u),(y, z)\}, \theta_{1}$ be the congruence generated by $\{(x, y),(z, u)\}$, and $\psi$ be the congruence generated 
by $\{(y, z)\}$. Applying part (1) to this situation, we have that

$$
(x, u) \in \psi+{ }^{\prime} \theta_{0} \theta_{1} \text {. }
$$

This means that $(x, u)$ belongs to $\operatorname{Cg}_{\mathscr{H}}\left(\{(y, z)\} \cup \theta_{0} \theta_{1}\right)$, and since $\mathrm{Cg}_{\mathscr{H}}$ is algebraic, there exists a finite set $T$ of ordered pairs contained in $\theta_{0} \theta_{1}$ such that $(x, u)$ belongs to $\operatorname{Cg}_{\mathscr{K}}(\{(y, z)\} \cup T)$. We can write

$$
T=\left\{\left\langle p^{\mathbf{F}}(x, y, z, u), q^{\mathbf{F}}(x, y, z, u)\right\rangle:\langle p, q\rangle \in \Sigma_{s}\right\}
$$

for a finite set $\Sigma_{s}$ of pairs of terms.

The equations in (2)(i) hold in $\mathscr{K}$ because $T$ is a subset of $\theta_{0} \theta_{1}$. The condition (2)(ii) holds because $(x, u)$ is in the $\mathscr{K}$-congruence of $\mathbf{F}$ generated by $T$ together with the pair $(y, z)$.

The two assertions of this theorem will be valid if $\mathscr{K}$ is a quasivariety that extends to some modular variety. Hence Example 1.3 shows that the assertions of the theorem do not characterize the family of relatively modular quasivarieties, and shows, in fact, that no set of assertions similar to these can characterize this family. (Compare this statement to Theorem 4.1.)

Theorem 2.2 (Relative cube lemma). Let $\mathscr{K}$ be a relatively modular quasivariety.

(1) Suppose that $\mathbf{A} \in \mathscr{K}$, that $\theta_{0}, \theta_{1}, \psi \in \operatorname{Con}_{\mathscr{K}} \mathbf{A}$, and that $a_{0}, \ldots, a_{3}$, $b_{0}, \ldots, b_{3}$ are elements of $\mathbf{A}$ satisfying $\left(a_{0}, a_{1}\right),\left(a_{2}, a_{3}\right),\left(b_{0}, b_{1}\right),\left(b_{2}, b_{3}\right) \in$ $\theta_{0}$ and $\left(a_{i}, b_{i}\right) \in \theta_{1}$ for $i \in\{0,1,2,3\}$. If $\left(a_{1}, a_{2}\right),\left(b_{1}, b_{2}\right),\left(b_{0}, b_{3}\right) \in \psi$ then $\left(a_{0}, a_{3}\right) \in \psi+{ }^{\prime} \theta_{0} \theta_{1}$.

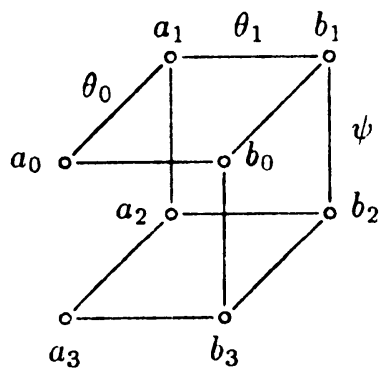

(2) There exists a finite set $\Sigma_{c}$ of ordered pairs of terms

$$
\left\langle p\left(x_{0}, \ldots, x_{3}, y_{0}, \ldots, y_{3}\right), q\left(x_{0}, \ldots, x_{3}, y_{0}, \ldots, y_{3}\right)\right\rangle
$$

in the language of $\mathscr{K}$ such that: (i) for each $\langle p, q\rangle \in \Sigma_{c}$ the equations

$$
\begin{aligned}
& p(x, y, y, x, z, u, u, z) \approx q(x, y, y, x, z, u, u, z), \\
& p(x, x, y, y, z, z, u, u) \approx q(x, x, y, y, z, z, u, u), \\
& p(x, y, z, u, x, y, z, u) \approx q(x, y, z, u, x, y, z, u)
\end{aligned}
$$

hold in $\mathscr{K}$; and (ii) if $\mathbf{A} \in \mathscr{K}$ and $a, d, u_{1}, u_{2}, u_{3} \in A$ and if

$$
p^{\mathbf{A}}\left(a, u_{1}, u_{2}, u_{3}, d, u_{1}, u_{2}, u_{3}\right)=q^{\mathbf{A}}\left(a, u_{1}, u_{2}, u_{3}, d, u_{1}, u_{2}, u_{3}\right)
$$

for all $\langle p, q\rangle \in \Sigma_{c}$, then $a=d$. 
Proof. Suppose that $\mathbf{A}, \theta_{0}, \theta_{1}, \psi$, and $a_{0}, \ldots, b_{3}$ satisfy the hypotheses of (1). Choose any pair of terms $\langle p, q\rangle$ in $\Sigma_{s}$. Define elements

$$
\begin{array}{ll}
a=p\left(a_{0}, a_{1}, a_{2}, a_{3}\right), & b=p\left(b_{0}, b_{1}, b_{2}, b_{3}\right), \\
d=q\left(a_{0}, a_{1}, a_{2}, a_{3}\right), & c=q\left(b_{0}, b_{1}, b_{2}, b_{3}\right) .
\end{array}
$$

Then the hypotheses of Theorem 2.1(1) are satisfied, and so $(a, d) \in \psi+{ }^{\prime} \theta_{0} \theta_{1}$. Also $\left(a_{1}, a_{2}\right) \in \psi+^{\prime} \theta_{0} \theta_{1}$. Now applying Theorem 2.1(2)(ii) in the algebra $\mathbf{A} / \gamma$, where $\gamma=\psi+{ }^{\prime} \theta_{0} \theta_{1}$, it follows that $\left(a_{0}, a_{3}\right) \in \gamma$ as desired.

Except for one small detail, statement (2) is a consequence of (1) in the same way that (2) followed from (1) in Theorem 2.1. The variation in the argument goes as follows. We will work in the free algebra $\mathbf{F} \in \mathscr{K}$ generated by $x_{0}, \ldots, x_{3}, y_{0}, \ldots, y_{3}$. Let $\psi$ be the congruence generated by $\left\{\left(x_{1}, y_{1}\right)\right.$, $\left.\left(x_{2}, y_{2}\right),\left(x_{3}, y_{3}\right)\right\}$ and let $\theta_{2}$ be the congruence generated by $\psi \cup\left\{\left(x_{0}, y_{0}\right)\right\}$. Let $\theta_{0}$ be the congruence generated by the pairs $\left\{\left(x_{0}, x_{3}\right),\left(x_{1}, x_{2}\right),\left(y_{0}, y_{3}\right)\right.$, $\left.\left(y_{1}, y_{2}\right)\right\}$ and $\theta_{1}$ be the congruence generated by $\left\{\left(x_{0}, x_{1}\right),\left(x_{2}, x_{3}\right),\left(y_{0}, y_{1}\right)\right.$, $\left.\left(y_{2}, y_{3}\right)\right\}$. All of these congruences are $\mathscr{K}$-congruences. Until this point the proof has been like that of Theorem 2.1. Now, an application of part (1) of this theorem shows that $\left(x_{0}, y_{0}\right) \in \psi+{ }^{\prime} \theta_{0} \theta_{1}$. Further, $\left(x_{0}, y_{0}\right) \in \theta_{2}$ and $\psi<\theta_{2}$. The relative modularity of $\mathscr{K}$ implies that $\left(x_{0}, y_{0}\right) \in \psi+{ }^{\prime} \theta_{0} \theta_{1} \theta_{2}$. From this point on the proof is exactly like the proof of Theorem 2.1 .

Definition 2.1. Given elements $a, b, c, d$ in an algebra $\mathbf{A}$, we define $Z(a, b ; c, d)$ to mean that for all polynomial operations $f(x, y)$ and $g(x, y)$ of $\mathbf{A}$, if

$$
f(a, c)=g(a, c), \quad f(\dot{a}, d)=g(a, d), \quad f(b, c)=g(b, c)
$$

then $f(b, d)=g(b, d)$. The relation $Z(a, b ; c, d)$ can be rendered as $(a, b)$ centralizes $(c, d)$ relative to the two-term condition.

Using the terms $\Sigma_{s}$ from the relative shifting lemma, we now show that in a relatively modular quasivariety the relation just defined is very well behaved.

Lemma 2.3. Suppose that $\mathscr{K}$ is relatively modular and $\mathbf{A} \in \mathscr{K}$.

(a) For any $a, b, c, d \in A$ we have

$$
Z(a, b ; c, d) \leftrightarrow Z(c, d ; a, b) \leftrightarrow Z(a, b ; d, c) .
$$

(b) For any $(a, b) \in A^{2}$ the relation

$$
Z(a, b)=\{(c, d): Z(a, b ; c, d)\}
$$

is a congruence of $\mathbf{A}$.

(c) Suppose that $Z\left(a_{i}, b_{i} ; c_{j}, d_{j}\right)$ for $0 \leq i<m$ and $0 \leq j<n$. Then if $f(\bar{x}, \bar{y})$ and $g(\bar{x}, \bar{y})$ are any $(m+n)$-ary polynomials of $\mathbf{A}$ such that $f(\bar{a}, \bar{c})=$ $g(\bar{a}, \bar{c}), f(\bar{a}, \bar{d})=g(\bar{a}, \bar{d})$, and $f(\bar{b}, \bar{c})=g(\bar{b}, \bar{c})$, it follows that $f(\bar{b}, \bar{d})=$ $g(\bar{b}, \bar{d})$.

Proof. Let $\Sigma_{s}$ be the finite set of pairs of terms supplied by Theorem 2.1. To prove statement (a), suppose that $Z(a, b ; c, d)$. It should be clear that this implies $Z(c, d ; a, b)$. To see that $Z(a, b ; d, c)$, let $f(x, y)$ and $g(x, y)$ be any two binary polynomial operations of $\mathbf{A}$ and suppose that $f(a, c)=$ 
$g(a, c), f(a, d)=g(a, d)$, and $f(b, d)=g(b, d)$. In order to prove that $f(b, c)=g(b, c)$, note that by Theorem 2.1 it will suffice to prove that for any $\langle p, q\rangle \in \Sigma_{s}$ we have

$$
\begin{aligned}
& p(f(b, c), f(b, d), g(b, d), g(b, c)) \\
& \quad=q(f(b, c), f(b, d), g(b, d), g(b, c)) .
\end{aligned}
$$

Given such a $p$ and $q$, define

$$
f^{\prime}(x, y)=p(f(x, c), f(x, y), g(x, y), g(x, c))
$$

and

$$
g^{\prime}(x, y)=q(f(x, c), f(x, y), g(x, y), g(x, c)) .
$$

From the equations satisfied by $p$ and $q$ in $\mathscr{K}$, it follows that we have $f^{\prime}(a, c)=g^{\prime}(a, c), f^{\prime}(a, d)=g^{\prime}(a, d)$, and $f^{\prime}(b, c)=g^{\prime}(b, c)$. Thus from $Z(a, b ; c, d)$ we conclude that $f^{\prime}(b, d)=g^{\prime}(b, d)$; and this is just what we had to prove.

To prove (b), let us first show that $Z(a, b)$ is transitive. (The relation is obviously reflexive, and by statement (a) it is symmetric.) Suppose that $(u, v),(v, w) \in Z(a, b)$; i.e., that $Z(a, b ; u, v)$ and $Z(a, b ; v, w)$. To see that $Z(a, b ; u, w)$, let $f(x, y)$ and $g(x, y)$ be polynomials such that $f$ and $g$ are equal at $(x, y)=(a, u),(a, w),(b, u)$. We prove that $f(b, w)$ $=g(b, w)$ by showing that for each $\langle p, q\rangle \in \Sigma_{s}, p$ and $q$ are equal at $(f(b, w), f(a, w), g(a, w), g(b, w))$, then applying Theorem 2.1 .

To do this consider the polynomials

$$
\begin{aligned}
f^{\prime}(x, y) & =p(f(x, y), f(a, y), g(a, y), g(x, y)), \\
g^{\prime}(x, y) & =q(f(x, y), f(a, y), g(a, y), g(x, y)) .
\end{aligned}
$$

Now $f^{\prime}(a, u)=g^{\prime}(a, u), f^{\prime}(a, v)=g^{\prime}(a, v)$, and $f^{\prime}(b, u)=g^{\prime}(b, u)$ from the equations valid for $p, q$; hence $f^{\prime}(b, v)=g^{\prime}(b, v)$ since $Z(a, b ; u, v)$. Now obviously, $f^{\prime}(a, y)=g^{\prime}(a, y)$ for any $y$; thus $f^{\prime}(b, v)=g^{\prime}(b, v)$ and $Z(a, b ; v, w)$ imply $f^{\prime}(b, w)=g^{\prime}(b, w)$. This is the equation claimed in the last paragraph. It completes the proof that $Z(a, b)$ is transitive.

Since $Z(a, b)$ is seen to be an equivalence relation, all that remains in order to conclude that $Z(a, b)$ is a congruence is to observe that it follows trivially from our definition that whenever $Z(a, b ; c, d)$ and $h(x)$ is a unary polynomial operation of $\mathbf{A}$ then $Z(a, b ; h(c), h(d))$. This means that $Z(a, b)$ is closed under unary polynomials; so it is a congruence.

Statement (c) can be proved by induction on $m$ for $n=1$, and then by induction on $n$. The case $m=n=1$ is a tautology. Now suppose the statement is true for a certain $m$ when $n=1$. Let $\bar{a}, \bar{b}$ be $(m+1)$-tuples, $c, d$ be elements, $f(\bar{x}, y), g(\bar{x}, y)$ be polynomials such that $Z\left(a_{i}, b_{i} ; c, d\right)$ holds for all $i \leq m$, and $f(\bar{a}, c)=g(\bar{a}, c), f(\bar{a}, d)=g(\bar{a}, d), f(\bar{b}, c)=$ $g(\bar{b}, c)$. As usual, letting $\langle p, q\rangle \in \Sigma_{s}$, we need to prove that $p$ and $q$ agree at $(f(\bar{b}, d), f(\bar{b}, c), g(\bar{b}, c), g(\bar{b}, d))$. We do this in two stages. Consider first the $(m+1)$-ary polynomials

$$
\begin{aligned}
f^{\prime}(\bar{x}, y) & =p\left(f\left(\bar{x}, a_{m}, y\right), f\left(\bar{x}, a_{m}, c\right), g\left(\bar{x}, a_{m}, c\right), g\left(\bar{x}, a_{m}, y\right)\right), \\
g^{\prime}(\bar{x}, y) & =q\left(f\left(\bar{x}, a_{m}, y\right), f\left(\bar{x}, a_{m}, c\right), g\left(\bar{x}, a_{m}, c\right), g\left(\bar{x}, a_{m}, y\right)\right) .
\end{aligned}
$$


Let $\bar{a}^{\prime}=\left(a_{0}, \ldots, a_{m-1}\right), \bar{b}^{\prime}=\left(b_{0}, \ldots, b_{m-1}\right)$. From our assumptions, we see that $f^{\prime}$ and $g^{\prime}$ agree at $\left(\bar{a}^{\prime}, c\right),\left(\bar{a}^{\prime}, d\right)$, and $\left(\bar{b}^{\prime}, c\right)$. Thus, by the induction assumption, we have $f^{\prime}$ and $g^{\prime}$ agreeing at $\left(b_{0}, \ldots, b_{m-1}, d\right)$. This means that where we define

$$
\begin{aligned}
& f^{\prime \prime}(x, y)=p\left(f\left(\bar{b}^{\prime}, x, y\right), f\left(\bar{b}^{\prime}, x, c\right), g\left(\bar{b}^{\prime}, x, c\right), g\left(\bar{b}^{\prime}, x, y\right)\right), \\
& g^{\prime \prime}(x, y)=q\left(f\left(\bar{b}^{\prime}, x, y\right), f\left(\bar{b}^{\prime}, x, c\right), g\left(\bar{b}^{\prime}, x, c\right), g\left(\bar{b}^{\prime}, x, y\right)\right),
\end{aligned}
$$

we have $f^{\prime \prime}\left(a_{m}, d\right)=g^{\prime \prime}\left(a_{m}, d\right)$. Since it also follows directly from our assumptions that $f^{\prime \prime}\left(a_{m}, c\right)=g^{\prime \prime}\left(a_{m}, c\right)$ and $f^{\prime \prime}\left(b_{m}, c\right)=g^{\prime \prime}\left(b_{m}, c\right)$, then from the fact that $Z\left(a_{m}, b_{m} ; c, d\right)$ we derive $f^{\prime \prime}\left(b_{m}, d\right)=g^{\prime \prime}\left(b_{m}, d\right)$. This is exactly the equation we were after. The inductive proof that (c) holds for $n=1$ and for any positive value of $m$ is now complete.

We complete the proof of (c) by assuming that the statement holds for a certain value of $n$ and for all positive $m$. Then let $\bar{a}, \bar{b}$ be $m$-tuples (for some $m>0)$, let $\bar{c}, \bar{d}$ be $(n+1)$-tuples, and suppose that $f$ and $g$ are $(m+n+1)$-ary polynomials and the hypotheses in (c) are fulfilled. Thus $f$ and $g$ agree at $(\bar{x}, \bar{y})=(\bar{a}, \bar{c}),(\bar{a}, \bar{d}),(\bar{b}, \bar{c})$. As usual, $\langle p, q\rangle$ denotes an arbitrary pair in $\Sigma_{s}$. Our reasoning parallels that of the last paragraph. We define $(m+n)$-ary polynomials $f^{\prime}$ and $g^{\prime}$ by

$$
\begin{gathered}
f^{\prime}(\bar{x}, \bar{y})=p\left(f\left(\bar{x} ; \bar{y}, c_{n}\right), f\left(\bar{a} ; \bar{y}, c_{n}\right), g\left(\bar{a} ; \bar{y}, c_{n}\right), g\left(\bar{x} ; \bar{y}, c_{n}\right)\right), \\
g^{\prime}(\bar{x}, \bar{y})=q\left(f\left(\bar{x} ; \bar{y}, c_{n}\right), f\left(\bar{a} ; \bar{y}, c_{n}\right), g\left(\bar{a} ; \bar{y}, c_{n}\right), g\left(\bar{x} ; \bar{y}, c_{n}\right)\right) .
\end{gathered}
$$

We note that $f^{\prime}$ and $g^{\prime}$ agree at $\left(\bar{a}, \bar{c}^{\prime}\right),\left(\bar{a}, \bar{d}^{\prime}\right)$, and $\left(\bar{b}, \bar{c}^{\prime}\right)$, where $\bar{c}^{\prime}$ and $\bar{d}^{\prime}$ are $\bar{c}$ and $\bar{d}$ truncated by removing the last term of these $(n+1)$-tuples. Using the inductive assumption, we conclude that where

$$
\begin{aligned}
& f^{\prime \prime}(\bar{x}, y)=p\left(f\left(\bar{x} ; \bar{d}^{\prime}, y\right), f\left(\bar{a} ; \bar{d}^{\prime}, y\right), g\left(\bar{a}, \bar{d}^{\prime}, y\right), g\left(\bar{x}, \bar{d}^{\prime}, y\right)\right), \\
& g^{\prime \prime}(\bar{x}, y)=q\left(f\left(\bar{x} ; \bar{d}^{\prime}, y\right), f\left(\bar{a} ; \bar{d}^{\prime}, y\right), g\left(\bar{a}, \bar{d}^{\prime}, y\right), g\left(\bar{x}, \bar{d}^{\prime}, y\right)\right),
\end{aligned}
$$

we have $f^{\prime \prime}\left(\bar{b}, c_{n}\right)=g^{\prime \prime}\left(\bar{b}, c_{n}\right)$. Since it is obvious that $f^{\prime \prime}$ and $g^{\prime \prime}$ agree at arguments of the form $(\bar{a}, y)$, it follows from statement (c) in the case $n=1$ (which we have already proved) that $f^{\prime \prime}\left(\bar{b}, d_{n}\right)=g^{\prime \prime}\left(\bar{b}, d_{n}\right)$. In other words, we have proved that $p$ and $q$ agree at $(f(\bar{b}, \bar{d}), f(\bar{a}, \bar{d}), g(\bar{a}, \bar{d}), g(\bar{b}, \bar{d}))$. As usual, since this holds for all $\langle p, q\rangle \in \Sigma_{s}$ we can conclude that $f(\bar{b}, \bar{d})=$ $g(\bar{b}, \bar{d})$, as desired.

Definition 2.2. Let $\alpha, \beta, \mu$ be congruences of an algebra $\mathbf{A}$. We write $Z(\alpha, \beta ; \mu)$ to denote that for all $(a, b) \in \alpha$ and $(c, d) \in \beta$ and for all binary polynomial operations $f(x, y)$ and $g(x, y)$ of $\mathbf{A}$, if $f(a, c) \mu g(a, c)$, $f(a, d) \mu g(a, d)$, and $f(b, c) \mu g(b, c)$ then $f(b, d) \mu g(b, d)$. If this relation holds, then we say that $\alpha$ centralizes $\beta$ modulo $\mu$ (in the sense of the two-term condition). The relation $Z\left(\alpha, \beta ; 0_{A}\right)$ will be abbreviated to $Z(\alpha, \beta)$.

Lemma 2.4. Let $\mathscr{K}$ be a relatively modular quasivariety, $\mathrm{A} \in \mathscr{K}, \alpha, \beta \in$ $\operatorname{Con} \mathbf{A}$, and $\mu \in \operatorname{Con}_{\mathscr{H}} \mathbf{A}$.

(1) If $Z(\alpha, \beta ; \mu)$ then $\alpha$ centralizes $\beta$ modulo $\mu$ in the sense of the classical one-term condition.

(2) We have $Z(\alpha, \beta)$ iff $Z(a, b ; c, d)$ holds for all $(a, b) \in \alpha$ and $(c, d)$ $\in \beta$.

(3) The conditions $Z(\alpha, \beta ; \mu)$ and $Z(\alpha+\mu, \beta+\mu ; \mu)$ are equivalent and equivalent to $Z(\bar{\alpha}, \bar{\beta})$ holding in $\mathbf{A} / \mu$, where $\bar{\alpha}=(\alpha+\mu) / \mu$ and $\bar{\beta}=(\beta+\mu) / \mu$. 
Proof. All parts are straightforward, using Lemma 2.3, except (1). To prove (1), suppose that $Z(\alpha, \beta ; \mu)$, that $f(x, \bar{y})$ is a polynomial operation of $\mathbf{A}$, and that $a, b, \bar{c}, \bar{d}$ are elements and tuples of elements of $\mathbf{A}$ such that $(a, b) \in \alpha$ and $\left(c_{i}, d_{i}\right) \in \beta$ for all $i$. Assume also that $f(a, \bar{c}) \equiv f(a, \bar{d})(\bmod \mu)$. We must prove that this equivalence modulo $\mu$ remains true after exchanging $a$ for $b$. To do it, let $\langle p, q\rangle$ be any member of $\Sigma_{s}$, and define

$$
\begin{aligned}
& f^{\prime}(x, \bar{y})=p(f(x, \bar{c}), f(a, \bar{c}), f(a, \bar{y}), f(x, \bar{y})), \\
& g^{\prime}(x, \bar{y})=q(f(x, \bar{c}), f(a, \bar{c}), f(a, \bar{y}), f(x, \bar{y})) .
\end{aligned}
$$

Then $f^{\prime}$ and $g^{\prime}$ give a pair of $\mu$-congruent values when applied to each of the tuples $(a, \bar{c}),(a, \bar{d}),(b, \bar{c})$. Hence by Lemma 2.3(c) applied in the algebra $\mathbf{A} / \mu$, we deduce that $f^{\prime}(b, \bar{d}) \equiv g^{\prime}(b, \bar{d})(\bmod \mu)$. This means that

$$
\begin{aligned}
& p(f(b, \bar{c}), f(a, \bar{c}), f(a, \bar{d}), f(b, \bar{d})) \\
& \quad \equiv q(f(b, \bar{c}), f(a, \bar{c}), f(a, \bar{d}), f(b, \bar{d}))(\bmod \mu) .
\end{aligned}
$$

Since $f(a, \bar{c})$ and $f(a, \bar{d})$ represent the same element of $\mathbf{A} / \mu$, applying Theorem 2.1 to the algebra $\mathbf{A} / \mu$ gives us that $f(b, \bar{c})$ and $f(b, \bar{d})$ are $\mu$ equivalent.

Henceforth, $\mathscr{K}$ denotes a fixed relatively modular quasivariety, and all algebras entering the discussion belong to $\mathscr{K}$. Our stroke notation for denoting the $\mathscr{K}$-extension of a congruence has been explained earlier. We reintroduce the convention of denoting the kernels of the coordinate projections from a subdirect product of two algebras by $\eta_{0}$ and $\eta_{1}$.

Definition 2.3. Suppose that $\mathbf{A} \in \mathscr{K}$ and $\alpha, \beta \in \operatorname{Con} \mathbf{A}$. By $\mathbf{A}(\alpha)$ we denote the subalgebra of $\mathbf{A}^{2}$ whose universe is $\alpha$. By $\Delta_{\alpha \beta}$ we denote the congruence on $\mathbf{A}(\alpha)$ generated by identifying $(x, x)$ and $(y, y)$ whenever $(x, y) \in \beta$ (i.e., the congruence on $\mathbf{A}(\alpha)$ generated by the $\beta$-diagonal). We define $[\alpha, \beta]$ to consist of the ordered pairs $(x, y)$ such that $(x, y)$ is $\Delta_{\alpha \beta}^{\prime}$-congruent to $(x, x)$. Notice that the set of congruences

$$
\left\{\theta \in \operatorname{Con} \mathbf{A}: \theta_{0} \Delta_{\alpha \beta}=\theta_{1} \Delta_{\alpha \beta} \text { holds in Con } \mathbf{A}(\alpha)\right\}
$$

is closed under arbitrary intersection. We let $\kappa(\alpha, \beta)$ denote the least member of this set.

Lemma 2.5. We have $\Delta_{\alpha \beta}^{\prime}=\Delta_{\alpha \beta^{\prime}}^{\prime} \leq \beta^{\prime} \times \beta^{\prime}$. Moreover, the following are equivalent for any $a, b \in \mathbf{A}$, and they imply $(a, b) \in \alpha \beta^{\prime}$.

(1) $(a, b) \in[\alpha, \beta]$.

(2) $(a, b) \Delta_{\alpha \beta}^{\prime}(c, c)$ for some $c$.

(3) $(a, c) \Delta_{\alpha \beta}^{\prime}(b, c)$ for some $c$.

(4) $(c, a) \Delta_{\alpha \beta}^{\prime}(c, b)$ for some $c$.

Proof. Clearly $\Delta_{\alpha \beta}^{\prime} \leq \beta^{\prime} \times \beta^{\prime}$ (so $[\alpha, \beta] \subseteq \alpha \beta^{\prime}$ ), and $\Delta_{\alpha \beta}^{\prime}$ is invariant under the canonical automorphism of $\mathbf{A}(\alpha)$, i.e.,

$$
(x, y) \Delta_{\alpha \beta}^{\prime}(u, v) \text { iff }(y, x) \Delta_{\alpha \beta}^{\prime}(v, u) .
$$

It is easy to show that $\Delta_{\alpha \beta}^{\prime}$ contains the $\beta^{\prime}$ diagonal on $\mathbf{A}(\alpha)$; from this it follows that $\Delta_{\alpha \beta}^{\prime}=\Delta_{\alpha \beta^{\prime}}^{\prime}$. These facts easily imply that (3) and (4) are equivalent, and that (1) and (2) are equivalent. It is easy to show that (1) implies (4). 
Finally, to see that (3) implies (2), suppose that $(a, c) \Delta_{\alpha \beta}^{\prime}(b, c)$. Then $a, c$, and $b$ are all $\alpha$-related. We have

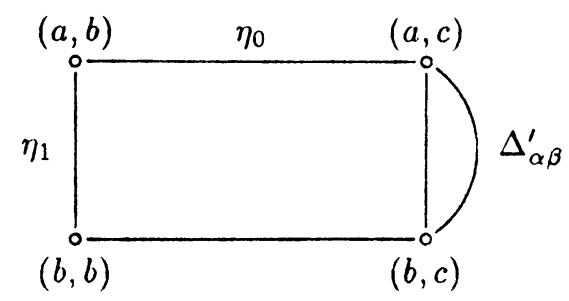

By the relative shifting lemma, $(a, b) \Delta_{\alpha \beta}^{\prime}(b, b)$.

Lemma 2.6. Let $\mathbf{A} \in \mathscr{K}$ and $\alpha, \beta \in \operatorname{Con} \mathbf{A}$.

(1) $[\alpha, \beta]$ is a congruence. If $\alpha \in \operatorname{Con}_{\mathscr{K}} \mathbf{A}$, then $[\alpha, \beta] \in \operatorname{Con}_{\mathscr{K}} \mathbf{A}$. If $\beta \in \operatorname{Con}_{\mathscr{K}} \mathbf{A}$, then $[\alpha, \beta] \leq \alpha \beta$.

(2) In $\operatorname{Con} \mathbf{A}(\alpha),[\alpha, \beta]_{0} \eta_{1}=\Delta_{\alpha \beta}^{\prime} \eta_{1},[\alpha, \beta]_{1} \eta_{0}=\Delta_{\alpha \beta}^{\prime} \eta_{0}$, and

$$
[\alpha, \beta]_{0} \Delta_{\alpha \beta}^{\prime}=[\alpha, \beta]_{1} \Delta_{\alpha \beta}^{\prime}=[\alpha, \beta]_{0}[\alpha, \beta]_{1} .
$$

(3) Any $\mu \in \operatorname{Con}_{\mathscr{K}} \mathbf{A}$ satisfies $\mu_{0} \Delta_{\alpha \beta}^{\prime}=\mu_{1} \Delta_{\alpha \beta}^{\prime}$ iff $\mu \geq \kappa(\alpha, \beta)$ iff $\mu \geq$ $[\alpha, \beta]$.

Proof. $[\alpha, \beta]$ is clearly reflexive and it is symmetric by (3) of Lemma 2.5. One can also use Lemma $2.5(3)$ to show that $[\alpha, \beta]$ has the substitution property. To see that it is transitive, suppose that $a[\alpha, \beta] b[\alpha, \beta] c$. Then

$$
(a, b) \Delta_{\alpha \beta}^{\prime}(b, b) \Delta_{\alpha \beta}^{\prime}(c, b) ;
$$

hence $(a, c) \in[\alpha, \beta]$ by Lemma 2.5. Thus the relation is a congruence. If $\beta$ $=\beta^{\prime}$ then, by Lemma 2.5, $[\alpha, \beta] \leq \alpha \beta^{\prime}=\alpha \beta$. Before proving that it is a $\mathscr{K}$-congruence if $\alpha$ is, we prove (2).

We have seen in Lemma 2.5 that $\Delta_{\alpha \beta}^{\prime} \eta_{1} \leq[\alpha, \beta]_{0} \eta_{1}$. On the other hand, if $(a, b) \in[\alpha, \beta]$ and $(a, c),(b, c) \in \mathbf{A}(\alpha)$ then we have the rectangle

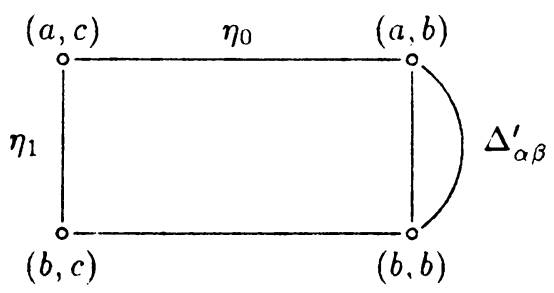

which yields, via the relative shifting lemma, that $(a, c) \Delta_{\alpha \beta}^{\prime}(b, c)$. Thus $[\alpha, \beta]_{0} \eta_{1}=\Delta_{\alpha \beta}^{\prime} \eta_{1}$. The similar equation with the subscripts 0 and 1 interchanged must of course also be true.

To see that $[\alpha, \beta]_{0}$ and $[\alpha, \beta]_{1}$ have the same intersection with $\Delta_{\alpha \beta}^{\prime}$, we apply the cube lemma. Suppose that $(a, b) \Delta_{\alpha \beta}^{\prime}(c, d)$ and $(a, c) \in[\alpha, \beta]$. We have a cube in $\mathbf{A}(\alpha)$ : 


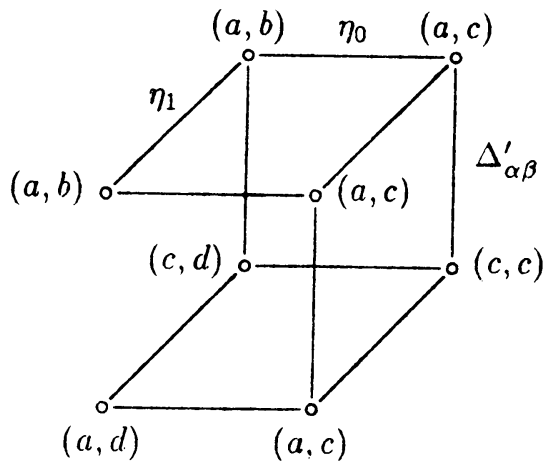

The cube lemma asserts that $(a, b)$ and $(a, d)$ must be congruent modulo $\Delta_{\alpha \beta}^{\prime}+\eta_{0} \eta_{1}$, i.e., modulo $\Delta_{\alpha \beta}^{\prime}$. Hence $(b, d) \in[\alpha, \beta]$. This shows that $[\alpha, \beta]_{0} \Delta_{\alpha \beta}^{\prime} \leq[\alpha, \beta]_{1}$. We thus conclude that $[\alpha, \beta]_{0} \Delta_{\alpha \beta}^{\prime}=[\alpha, \beta]_{1} \Delta_{\alpha \beta}^{\prime}$. These equal congruences are obviously contained in $[\alpha, \beta]_{0}[\alpha, \beta]_{1}$. For the reverse inclusion, we simply note (by examining elements) that

$$
\begin{aligned}
{[\alpha, \beta]_{0}[\alpha, \beta]_{1} } & =[\alpha, \beta]_{0} \eta_{1}+[\alpha, \beta]_{1} \eta_{0} \\
& =\Delta_{\alpha \beta}^{\prime} \eta_{1}+\Delta_{\alpha \beta}^{\prime} \eta_{0} \leq \Delta_{\alpha \beta}^{\prime}[\alpha, \beta]_{1}+\Delta_{\alpha \beta}^{\prime}[\alpha, \beta]_{0} .
\end{aligned}
$$

Now we return to the second statement in (1). By (2), we have $[\alpha, \beta]_{0} \eta_{1}=$ $\Delta_{\alpha \beta}^{\prime} \eta_{1}$. Applying the prime homomorphism (see Theorem 1.1) we get that $[\alpha, \beta]_{0}^{\prime} \eta_{1}=\Delta_{\alpha \beta}^{\prime} \eta_{1}$. Hence, $[\alpha, \beta]_{0} \eta_{1}=\Delta_{\alpha \beta}^{\prime} \eta_{1}=[\alpha, \beta]_{0}^{\prime} \eta_{1}$. Forming meets on both sides with $\alpha_{0}$, we find that $(\alpha[\alpha, \beta])_{0} \eta_{1}=\left(\alpha[\alpha, \beta]^{\prime}\right)_{0} \eta_{1}$. But in $\operatorname{Con} \mathbf{A}(\alpha)$, whenever $\theta_{0} \leq \tilde{\theta}_{0} \leq \alpha_{0}$ and $\theta_{0} \eta_{1}=\tilde{\theta}_{0} \eta_{1}$ holds, one always has $\theta=\tilde{\theta}$. Hence, in Con $\mathbf{A}$ we have $\alpha[\alpha, \beta]^{\prime}=\alpha[\alpha, \beta]=[\alpha, \beta]$. Now $[\alpha, \beta] \leq \alpha$, so $[\alpha, \beta]^{\prime} \leq \alpha^{\prime}$. If $\alpha=\alpha^{\prime}$, then $[\alpha, \beta]=\alpha[\alpha, \beta]^{\prime}=\alpha^{\prime}[\alpha, \beta]^{\prime}$ $=[\alpha, \beta]^{\prime}$.

To prove (3), notice that if $\mu \in \operatorname{Con}_{\mathscr{K}} \mathbf{A}$, then $\mu_{0} \Delta_{\alpha \beta}^{\prime}=\mu_{1} \Delta_{\alpha \beta}^{\prime}$ implies $\mu_{0} \Delta_{\alpha \beta}=\mu_{1} \Delta_{\alpha \beta}$ since the latter equation is obtained from the former by taking meets with $\Delta_{\alpha \beta}$ on both sides. Conversely, we have that $\mu_{0} \Delta_{\alpha \beta}=\mu_{1} \Delta_{\alpha \beta}$ implies $\mu_{0} \Delta_{\alpha \beta}^{\prime}=\mu_{1} \Delta_{\alpha \beta}^{\prime}$ by applying the prime homomorphism. Thus, $\mu_{0} \Delta_{\alpha \beta}^{\prime}=\mu_{1} \Delta_{\alpha \beta}^{\prime}$ holds iff $\mu_{0} \Delta_{\alpha \beta}=\mu_{1} \Delta_{\alpha \beta}$ holds, and these equations obviously imply that $\kappa(\alpha, \beta) \leq \mu$ holds. The latter inclusion implies that $\mu \geq[\alpha, \beta]$, since by (2),

$$
\mu_{0} \Delta_{\alpha \beta}^{\prime} \geq \kappa(\alpha, \beta)_{0} \Delta_{\alpha \beta}^{\prime}=\kappa(\alpha, \beta)_{1} \Delta_{\alpha \beta}^{\prime} \geq \eta_{1} \Delta_{\alpha \beta}^{\prime}=[\alpha, \beta]_{0} \eta_{1} .
$$

Now assume that $\mu \in \operatorname{Con}_{\mathscr{K}} \mathbf{A}$ and $\mu \geq[\alpha, \beta]$. Suppose $(a, b) \mu_{0} \Delta_{\alpha \beta}^{\prime}(c, d)$. Then we have a rectangle

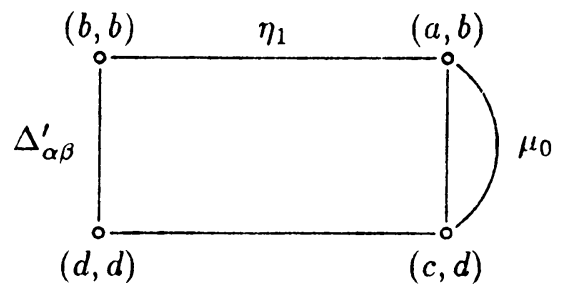


from which we get that $(b, b)$ and $(d, d)$ are congruent modulo

$$
\Delta_{\alpha \beta}^{\prime}\left(\eta_{1}+{ }^{\prime} \mu_{0} \Delta_{\alpha \beta}^{\prime}\right)=\Delta_{\alpha \beta}^{\prime} \eta_{1}+^{\prime} \mu_{0} \Delta_{\alpha \beta}^{\prime}=[\alpha, \beta]_{0} \eta_{1}+{ }^{\prime} \mu_{0} \Delta_{\alpha \beta}^{\prime} \leq \mu_{0} .
$$

This implies that $(b, d) \in \mu$, and hence $(a, b) \equiv(c, d)\left(\bmod \mu_{1}\right)$. Obviously, it follows that $\mu_{0} \Delta_{\alpha \beta}^{\prime}=\mu_{1} \Delta_{\alpha \beta}^{\prime}$.

Example 2.7. We examine the $\mathscr{K}$-commutator in a relatively distributive quasivariety $\mathscr{K}$. Since $\Delta_{\alpha \beta}^{\prime}=\Delta_{\alpha \beta^{\prime}}^{\prime} \leq \beta^{\prime} \times \beta^{\prime}$, it follows that in $\operatorname{Con} \mathbf{A}(\alpha)$ we have $\beta_{i}^{\prime}=\eta_{i}+\Delta_{\alpha \beta}^{\prime}=\eta_{i}+{ }^{\prime} \Delta_{\alpha \beta}^{\prime}$ for $i=0,1$. Hence,

$$
\beta_{0}^{\prime} \beta_{1}^{\prime}=\left(\eta_{0}+{ }^{\prime} \Delta_{\alpha \beta}^{\prime}\right)\left(\eta_{1}+{ }^{\prime} \Delta_{\alpha \beta}^{\prime}\right)=\eta_{0} \eta_{1}+{ }^{\prime} \Delta_{\alpha \beta}^{\prime}=\Delta_{\alpha \beta}^{\prime}
$$

Thus, $[\alpha, \beta]$ consists of the set of pairs $(x, y)$ for which $(x, y)$ is $\beta_{0}^{\prime} \beta_{1}^{\prime}$ related to $(x, x)$. This set of pairs is precisely $\alpha \beta^{\prime}$. Hence, $[\alpha, \beta]=\alpha \beta^{\prime}$ is a commutator equation holding in $\mathscr{K}$. Now suppose that some algebra in $\mathscr{K}$ has a congruence $\alpha$ that is not a $\mathscr{K}$-congruence. Then $\left[\alpha, \alpha^{\prime}\right]=\alpha \neq$ $\alpha^{\prime}=\left[\alpha^{\prime}, \alpha\right]$, so the $\mathscr{K}$-commutator of ordinary congruences is not always symmetric. Further, $\left[\alpha^{\prime}, \alpha\right]=\alpha^{\prime} \mathbb{Z} \alpha=\alpha^{\prime} \alpha$, so the $\mathscr{K}$-commutator of ordinary congruences is not always sub-multiplicative. Also, since $\left[\alpha, \alpha^{\prime}\right]=\alpha$, the $\mathscr{K}$-commutator of two ordinary congruences need not be a $\mathscr{K}$-congruence.

Even if $\alpha, \beta \in \operatorname{Con}_{\mathscr{K}} \mathbf{A}$, we may have $\Delta_{\alpha \beta} \neq \Delta_{\alpha \beta}^{\prime}$. In fact, the set of pairs $\Delta_{\alpha \beta}$-related to the diagonal in $\mathbf{A}(\alpha)$ may be different than the set of pairs $\Delta_{\alpha \beta^{-}}^{\prime}$ related to the diagonal. Let $\mathscr{K}$ be the class of all commutative rings $\mathbf{R}$ such that in $\mathbf{R}, x^{2}=0$ implies $x=0$. This class is a relatively distributive quasivariety. Con ${ }_{\mathscr{K}} \mathbf{R}$ consists of the congruences corresponding to the radical ideals of $\mathbf{R}$. For any $\mathbf{F} \in \mathscr{K}$ we also have $\mathbf{F}[x] \in \mathscr{K}$. Let $\alpha=\beta$ be the congruence on $\mathbf{F}[x]$ corresponding to the radical ideal generated by the element $x$. One can check that, in this example, $\langle(x, 0),(0,0)\rangle \in \Delta_{\alpha \beta}^{\prime}-\Delta_{\alpha \beta}$. Our claims follow from this.

Lemma 2.8. Suppose that $\alpha \leq \delta$ and $\beta \leq \gamma$. Then $\Delta_{\alpha \beta} \subseteq \Delta_{\delta \gamma}, \Delta_{\alpha \beta}^{\prime} \subseteq \Delta_{\delta \gamma}^{\prime}$, and $[\alpha, \beta] \leq[\delta, \gamma]$.

Proof. Straightforward.

We have proved that $[\alpha, \beta]$, as a binary operation on $\operatorname{Con}_{\mathscr{K}} \mathbf{A}$, is submultiplicative and monotone. Our next goal is to prove that this operation on $\operatorname{Con}_{\mathscr{K}} \mathbf{A}$ is symmetric and completely additive in each variable. In order to do so, we proceed to establish a connection between the commutator and the "centralizer" notion defined in Definitions 2.1 and 2.2.

Lemma 2.9. Suppose that $\alpha, \beta \in \operatorname{Con} \mathbf{A}$ and $\mu \in \operatorname{Con}_{\mathscr{K}} \mathbf{A}$. Then $Z(\alpha, \beta ; \mu)$ iff $\mu \geq[\alpha, \beta]$.

Proof. Assume first that $\mu \geq[\alpha, \beta]$, so that $\mu_{0} \Delta_{\alpha \beta}^{\prime}=\mu_{1} \Delta_{\alpha \beta}^{\prime}$ (by Lemma $2.6(3))$. In order to prove that $Z(\alpha, \beta ; \mu)$ holds, let $f(x, y)$ and $g(x, y)$ be polynomials of $\mathbf{A}$, let $(a, b) \in \alpha$ and $(c, d) \in \beta$, and suppose that $f(x, y) \equiv g(x, y)(\bmod \mu)$ for $(x, y)=(a, c),(a, d),(b, c)$. Note that the pairs

$$
\begin{array}{ll}
x_{0}=(f(a, d), f(b, d)), & x_{1}=(f(a, c), f(b, c)), \\
x_{2}=(g(a, c), g(b, c)), & x_{3}=(g(a, d), g(b, d))
\end{array}
$$


belong to $\mathbf{A}(\alpha)$ and that $x_{0} \Delta_{\alpha \beta}^{\prime} x_{1}$ and $x_{2} \Delta_{\alpha \beta}^{\prime} x_{3}$. Moreover, $\left(x_{1}, x_{2}\right) \in \mu_{0} \mu_{1}$ and $\left(x_{0}, x_{3}\right) \in \mu_{0}$. Therefore,

$$
\left(x_{0}, x_{3}\right) \in \mu_{0}\left(\Delta_{\alpha \beta}^{\prime}+^{\prime} \mu_{0} \mu_{1}\right)=\mu_{0} \Delta_{\alpha \beta}^{\prime}+^{\prime} \mu_{0} \mu_{1} \leq \mu_{1},
$$

since $\mu_{0} \Delta_{\alpha \beta}^{\prime} \leq \mu_{1}$. This simply means that $f(b, d) \equiv g(b, d)(\bmod \mu)$, which completes the proof that $Z(\alpha, \beta ; \mu)$.

Now suppose, conversely, that $Z(\alpha, \beta ; \mu)$. To see that $\mu \geq[\alpha, \beta]$ will require our first (rather nontrivial) use of the terms supplied by the relative cube lemma (Theorem 2.2). Using those terms, we shall show that $\mu_{0} \Delta_{\alpha \beta} \leq \mu_{1}$. Then applying primes we shall have that $\mu_{0} \Delta_{\alpha \beta}^{\prime} \leq \mu_{1}$, and an application of Lemma 2.6 then completes the proof. To simplify matters, we write $\Delta$ for $\Delta_{\alpha \beta}$.

First we prove

(10.1) Let $\left(a, a^{\prime}\right) \Delta\left(b, b^{\prime}\right)$ and let $f(x, y)$ and $g(x, y)$ be polynomials. If $f(a, a) \mu g(a, a), f\left(a^{\prime}, a^{\prime}\right) \mu g\left(a^{\prime}, a^{\prime}\right)$, and $f(a, b) \mu$ $g(a, b)$, then $f\left(a^{\prime}, b^{\prime}\right) \mu g\left(a^{\prime}, b^{\prime}\right)$.

To prove (10.1), assume its hypotheses. Since $\left(a, a^{\prime}\right) \Delta\left(b, b^{\prime}\right)$, we can choose elements

$$
a=x_{0}, x_{1}, \ldots, x_{n}=b, \quad a^{\prime}=y_{0}, y_{1}, \ldots, y_{n}=b^{\prime},
$$

pairs $\left(c_{i}, d_{i}\right) \in \beta$ for $i<n$, pairs of tuples $\left(\bar{u}_{i}, \bar{v}_{i}\right) \in \alpha^{m}$ for $i<n$ (and for a sufficiently large integer $m$ ), and polynomials $F_{i}$ so that, for each $i<n$,

$$
\begin{aligned}
\left(x_{i}, y_{i}\right) & =\left(F_{i}\left(\bar{u}_{i}, c_{i}\right), F_{i}\left(\bar{v}_{i}, c_{i}\right)\right), \\
\left(x_{i+1}, y_{i+1}\right) & =\left(F_{i}\left(\bar{u}_{i}, d_{i}\right), F_{i}\left(\bar{v}_{i}, d_{i}\right)\right) .
\end{aligned}
$$

In proving that $f\left(a^{\prime}, b^{\prime}\right) \mu g\left(a^{\prime}, b^{\prime}\right)$ we shall apply Theorem 2.2(2) in the algebra $\mathbf{A} / \mu$. To that end, let $\langle p, q\rangle$ be any member of $\Sigma_{c}$. Define, for $i \leq n$,

$$
\begin{gathered}
z_{i}=p\left(f\left(a^{\prime}, y_{i}\right), f\left(a, x_{i}\right), f\left(a^{\prime}, a^{\prime}\right), f(a, a),\right. \\
\left.g\left(a^{\prime}, y_{i}\right), g\left(a, x_{i}\right), g\left(a^{\prime}, a^{\prime}\right), g(a, a)\right), \\
w_{i}=q\left(f\left(a^{\prime}, y_{i}\right), f\left(a, x_{i}\right), f\left(a^{\prime}, a^{\prime}\right), f(a, a),\right. \\
\left.g\left(a^{\prime}, y_{i}\right), g\left(a, x_{i}\right), g\left(a^{\prime}, a^{\prime}\right), g(a, a)\right) .
\end{gathered}
$$

We will show that

$z_{0} \mu w_{0}$; moreover, for each $i<n, z_{i} \mu w_{i}$ iff $z_{i+1} \mu w_{i+1}$.

That $z_{0} \mu w_{0}$ follows from the third of the three equations in 2(i) of Theorem 2.2 satisfied by $p$ and $q$ in $\mathscr{K}$, since

$$
f\left(a^{\prime}, a^{\prime}\right) \mu g\left(a^{\prime}, a^{\prime}\right) \text { and } f(a, a) \mu g(a, a) .
$$

To show that $\left(z_{i}, w_{i}\right) \in \mu$ iff $\left(z_{i+1}, w_{i+1}\right) \in \mu$ we define two new polynomials. Put

$$
\begin{array}{r}
f^{\prime}(x, \bar{y}, z)=p\left(f\left(x, F_{i}(\bar{y}, z)\right), f\left(a, F_{i}\left(\bar{u}_{i}, z\right)\right), f(x, x), f(a, a),\right. \\
\left.g\left(x, F_{i}(\bar{y}, z)\right), g\left(a, F_{i}\left(\bar{u}_{i}, z\right)\right), g(x, x), g(a, a)\right), \\
g^{\prime}(x, \bar{y}, z)=q\left(f\left(x, F_{i}(\bar{y}, z)\right), f\left(a, F_{i}\left(\bar{u}_{i}, z\right)\right), f(x, x), f(a, a),\right. \\
\left.g\left(x, F_{i}(\bar{y}, z)\right), g\left(a, F_{i}\left(\bar{u}_{i}, z\right)\right), g(x, x), g(a, a)\right) .
\end{array}
$$


Now $f^{\prime}\left(a, \bar{u}_{i}, z\right)=g^{\prime}\left(a, \bar{u}_{i}, z\right)$ for every $z$, by the second of the three equations for $p$ and $q$. Hence by Lemma 2.3(c), applied in $\mathbf{A} / \mu$, and using that $Z(\alpha, \beta ; \mu)$, we must have

$$
f^{\prime}\left(a^{\prime}, \bar{v}_{i}, c_{i}\right) \mu g^{\prime}\left(a^{\prime}, \bar{v}_{i}, c_{i}\right) \leftrightarrow f^{\prime}\left(a^{\prime}, \bar{v}_{i}, d_{i}\right) \mu g^{\prime}\left(a^{\prime}, \bar{v}_{i}, d_{i}\right) .
$$

This equivalence can also be written as

$$
z_{i} \mu w_{i} \leftrightarrow z_{i+1} \mu w_{i+1},
$$

which finishes our proof of (10.2).

Now (10.2) implies that $z_{n} \mu w_{n}$. Since

$$
\begin{gathered}
z_{n}=p\left(f\left(a^{\prime}, b^{\prime}\right), f(a, b), f\left(a^{\prime}, a^{\prime}\right), f(a, a),\right. \\
\left.g\left(a^{\prime}, b^{\prime}\right), g(a, b), g\left(a^{\prime}, a^{\prime}\right), g(a, a)\right) \\
w_{n}=q\left(f\left(a^{\prime}, b^{\prime}\right), f(a, b), f\left(a^{\prime}, a^{\prime}\right), f(a, a),\right. \\
\left.g\left(a^{\prime}, b^{\prime}\right), g(a, b), g\left(a^{\prime}, a^{\prime}\right), g(a, a)\right),
\end{gathered}
$$

and since $\langle p, q\rangle \in \Sigma_{c}$ is arbitrary, it now follows by Theorem 2.2(2)(ii) that $f\left(a^{\prime}, b^{\prime}\right) \mu g\left(a^{\prime}, b^{\prime}\right)$, finishing our proof of (10.1).

To finish our proof that $\mu_{0} \Delta_{\alpha \beta} \leq \mu_{1}$, let $\left(a, a^{\prime}\right) \Delta_{\alpha \beta}\left(b, b^{\prime}\right)$ and $a \mu b$. Let $\langle p, q\rangle$ be any member of $\Sigma_{s}$. Put $f(x, y)=p(x, a, b, y)$ and $g(x, y)=$ $q(x, a, b, y)$. Then for any $x$ we have

$$
f(x, x) \mu p(x, a, a, x)=q(x, a, a, x) \mu g(x, x) .
$$

Thus, $f(a, a) \mu g(a, a)$ and $f\left(a^{\prime}, a^{\prime}\right) \mu g\left(a^{\prime}, a^{\prime}\right)$ holds. Moreover, $f(a, b)=$ $g(a, b)$. Thus, by $(10.1), f\left(a^{\prime}, b^{\prime}\right) \mu g\left(a^{\prime}, b^{\prime}\right)$; i.e.,

$$
p\left(a^{\prime}, a, a, b^{\prime}\right) \mu f\left(a^{\prime}, b^{\prime}\right) \mu g\left(a^{\prime}, b^{\prime}\right) \mu q\left(a^{\prime}, a, a, b^{\prime}\right) .
$$

Since this holds for all $\langle p, q\rangle \in \Sigma_{s}$, we conclude by Theorem 2.2(2)(ii) that $a^{\prime} \mu b^{\prime}$.

Lemma 2.10. Let $\alpha, \beta \in \operatorname{Con} \mathbf{A}$ and $\mu \in \operatorname{Con}_{\mathscr{K}} \mathbf{A}$. Then $[\alpha, \beta]^{\prime}=\left[\alpha^{\prime}, \beta^{\prime}\right]$; in fact, the following are equivalent.

(1) $Z(\alpha, \beta ; \mu)$.

(2) $Z\left(\alpha^{\prime}, \beta^{\prime} ; \mu\right)$.

(3) $\left[\alpha^{\prime}, \beta^{\prime}\right] \leq \mu$.

Proof. By Lemma 2.9, (2) and (3) are equivalent, and obviously (2) implies (1). Now suppose that (1) holds. By Lemmas 2.9 and 2.6(3), we have

$$
\mu_{0} \Delta_{\alpha \beta}^{\prime}=\mu_{1} \Delta_{\alpha \beta}^{\prime} \text {. }
$$

Since $\Delta_{\alpha \beta}^{\prime}=\Delta_{\alpha \beta^{\prime}}^{\prime}$, the displayed formula implies that $Z\left(\alpha, \beta^{\prime} ; \mu\right)$, again by Lemmas 2.9 and 2.6(3). Then by Lemma $2.3(\mathrm{a})$, we also have $Z\left(\beta^{\prime}, \alpha ; \mu\right)$. Now just as above, this implies $Z\left(\beta^{\prime}, \alpha^{\prime} ; \mu\right)$ and then $Z\left(\alpha^{\prime}, \beta^{\prime} ; \mu\right)$.

Corollary 2.11. For any $a, b \in \mathbf{A}$ the congruence $Z(a, b)$ of Definition 2.1 belongs to $\operatorname{Con}_{\mathscr{K}} \mathbf{A}$. In fact, the following are equivalent for any $a, b, c, d$ and, for $\theta(a, b)$ and $\theta(c, d)$, the ordinary congruences generated by the pairs $(a, b)$ and $(c, d)$.
(1) $Z(a, b ; c, d)$.
(2) $Z\left(\theta(a, b)^{\prime}, \theta(c, d)^{\prime}\right)$.
(3) $\left[\theta(a, b)^{\prime}, \theta(c, d)^{\prime}\right]=0_{A}$. 
Proof. Straightforward, from the preceding results.

Notice that the commutator operation (on Con $\mathbf{A}$ and on $\operatorname{Con}_{\mathscr{K}} \mathbf{A}$ ) that we have introduced seems to be dependent upon the specific relatively modular quasivariety containing $\mathbf{A}$ that is under consideration. This is because the passage from $\Delta_{\alpha \beta}$ to $\Delta_{\alpha \beta}^{\prime}$ depends upon $\mathscr{K}$. Thus we should call it the $\mathscr{K}$-commutator. Although the value of the $\mathscr{K}$-commutator of $\alpha, \beta \in$ Con $\mathbf{A}$ depends on $\mathscr{K}$, this value is closely related to $\kappa(\alpha, \beta)$ which is independent of $\mathscr{K}$. For example, we know from Lemma 2.6 that $\kappa(\alpha, \beta) \leq[\alpha, \beta]$ and that $\kappa(\alpha, \beta)^{\prime}=[\alpha, \beta]^{\prime}$. These relations imply a certain stability in the values of the relative commutator, which is emphasized in the next result.

Theorem 2.12. If $\mathscr{L} \subseteq \mathscr{K}$ are relatively modular quasivarieties and $\mathbf{A} \in \mathscr{L}$, then $\mathrm{Cg}_{\mathscr{L}}: \operatorname{Con}_{\mathscr{H}} \mathbf{A} \rightarrow \mathrm{Con}_{\mathscr{L}} \mathbf{A}$ is a commutator-preserving lattice homomorphism.

Proof. The fact that this homomorphism is a (join-complete) lattice homomorphism follows from Theorem 1.1. We only need to show that if $\alpha, \beta \in \operatorname{Con}_{\mathscr{K}} \mathbf{A}$, then $\operatorname{Cg}_{\mathscr{L}}\left([\alpha, \beta]_{\mathscr{K}}\right)=\left[\operatorname{Cg}_{\mathscr{L}}(\alpha), \operatorname{Cg}_{\mathscr{L}}(\beta)\right]_{\mathscr{L}}$. In fact, for any $\alpha, \beta \in \operatorname{Con} \mathbf{A}$ we have

$$
\begin{aligned}
\operatorname{Cg}_{\mathscr{L}}\left([\alpha, \beta]_{\mathscr{K}}\right) & =\operatorname{Cg}_{\mathscr{L}}\left(\mathrm{Cg}_{\mathscr{H}}(\kappa(\alpha, \beta))\right)=\mathrm{Cg}_{\mathscr{L}}(\kappa(\alpha, \beta)) \\
& =\operatorname{Cg}_{\mathscr{L}}\left([\alpha, \beta]_{\mathscr{L}}\right)=\left[\operatorname{Cg}_{\mathscr{L}}(\alpha), \operatorname{Cg}_{\mathscr{L}}(\beta)\right]_{\mathscr{L}} .
\end{aligned}
$$

Theorem 2.13. Let $\mathscr{K}$ be a relatively modular quasivariety and let $\mathbf{A}, \mathbf{B} \in \mathscr{K}$. For $\alpha, \beta, \beta_{i}(i \in I), \mu$ in $\operatorname{Con}_{\mathscr{K}} \mathbf{A}$, and $\delta, \theta$ in $\operatorname{Con}_{\mathscr{K}} \mathbf{B}$ the $\mathscr{K}$-commutator satisfies the following.

(1) $[\alpha, \beta]=[\beta, \alpha] \leq \alpha \beta$.

(2) $\left[\alpha, \bigvee^{\prime}\left\{\beta_{i}: i \in I\right\}\right]=\bigvee^{\prime}\left\{\left[\alpha, \beta_{i}\right]: i \in I\right\}$.

(3) $[\alpha, \beta] \leq \mu$ iff $\alpha$ centralizes $\beta$ modulo $\mu$ in the sense of the two-term condition.

(4) If $f: \mathbf{A} \rightarrow \mathbf{B}$ is onto, then $f^{-1}([\delta, \theta])=\operatorname{ker} f+^{\prime}\left[f^{-1}(\delta), f^{-1}(\theta)\right]$.

Proof. Parts (1) and (3) have already been proved. Part (4) follows directly from Lemmas 2.4 and 2.9. The only part of this theorem remaining to be proved is (2). Since the commutator is monotone, it is clear that the $\mathscr{K}$-congruence represented by the right-hand side of (2) is contained in the one represented by the left-hand side. To demonstrate the other inclusion, let $\mu$ be the smaller of these two congruences. By Lemma 2.10 , we have that $Z\left(\alpha, \beta_{i} ; \mu\right)$ for all $i$. It follows easily from Lemmas 2.3 and 2.4 that the set of all pairs $(c, d)$ such that $Z(a / \mu, b / \mu ; c / \mu, d / \mu)$ holds for all $(a, b) \in \alpha$ is a congruence $\delta$ of $\mathbf{A}$ and we have $Z(\alpha, \delta ; \mu)$ and $\beta_{i} \leq \delta$ for all $i$. Then $\delta=\delta^{\prime}$ follows from Lemma 2.10 , and so $\bigvee^{\prime}\left\{\beta_{i}: i \in I\right\} \leq \delta$ and $Z\left(\alpha, \bigvee^{\prime}\left\{\beta_{i}: i \in I\right\} ; \mu\right)$. Thus $\mu \geq\left[\alpha, \bigvee^{\prime}\left\{\beta_{i}: i \in I\right\}\right]$, which is precisely what we had to prove.

This ends our development of a basic commutator theory for relatively modular quasivarieties. Many of the results of commutator theory for modular varieties extend immediately to relatively modular quasivarieties. In the cases where this can be done, Theorem 1.1 and Theorem 2.13 are the tools needed to convert the proofs. On the other hand, there are some very important results in varietal commutator theory that we have not been able to extend to quasivarieties. Most importantly, we do not have a structure theorem for Abelian algebras. For modular varieties, it is known that an Abelian algebra is affine; 
that is, it is polynomially equivalent to a module over some ring. It can be shown that any quasivariety of cancellative, commutative semigroups with operators, which are endomorphisms for the semigroup structure, is relatively modular and consists of Abelian algebras. These algebras need not be affine, but they are quasi-affine. Another important problem that we have not solved is whether there is a connection between solvability and permuting congruences analogous to the results discovered by $\mathrm{H}$. P. Gumm for modular varieties. These are probably the most important problems in the theory that need to be solved.

\section{AN APPLication}

We mention one result of varietal commutator theory that does extend to quasivarieties.

Theorem 3.1. If $\mathscr{K}$ is relatively modular and there is a bound on the size of the relatively subdirectly irreducible algebras in $\mathscr{K}$, then $\mathscr{K} \models \mathscr{K}$-con $[\alpha \beta, \beta]=$ $\alpha[\beta, \beta]$. In particular, this will be true if $\mathscr{K}$ is finitely generated.

An algebra in $\mathscr{K}$ is relatively subdirectly irreducible if its $\mathscr{K}$-congruence lattice is monolithic (i.e., has a smallest nonzero member, which is called the monolith). We use $\mathscr{K}_{\mathrm{RSI}}$ to denote the class of algebras in $\mathscr{K}$ that are relatively subdirectly irreducible (relative to $\mathscr{K}$ ). If $\mathbf{A} \in \mathscr{K}_{\mathrm{RSI}}$ then the full congruence lattice of $\mathbf{A}$ need not be monolithic, but, if $\mathscr{K}$ satisfies the extension principle, then the zero element of this lattice must be at least meet-irreducible. We say that such an algebra (whose zero congruence is a meet-irreducible element of the congruence lattice) is finitely subdirectly irreducible; and we write $\mathscr{K}_{\mathrm{FSI}}$ for the class of algebras in $\mathscr{K}$ that are finitely subdirectly irreducible. Thus, we have $\mathscr{K}_{\text {RSI }} \subseteq \mathscr{K}_{\text {FSI }}$ whenever $\mathscr{K}$ is a quasivariety satisfying the extension principle. We use $\mathscr{K}_{\text {SI }}$ for the class of algebras in $\mathscr{K}$ that are subdirectly irreducible in the absolute sense (i.e., whose full congruence lattice is monolithic).

The first statement of Theorem 3.1 can be proved in essentially the same way as Theorem 10.14 in [FM], which is the corresponding statement for modular varieties. The second statement follows from the the first statement and the fact that when $\mathscr{K}$ is generated by the class of algebras $M$, then $\mathscr{K}_{\mathrm{FSI}} \subseteq \boldsymbol{S P}_{u}(M)$; hence, if $\mathscr{K}$ is relatively modular and if $M$ is a finite set of finite algebras, then $\mathscr{K}_{\mathrm{RSI}} \subseteq \mathscr{K}_{\mathrm{FSI}} \subseteq \boldsymbol{S}(M)$.

Notice that the relative commutator equation in Theorem 3.1 may be written as an implication that holds for all congruences, not just $\mathscr{K}$-congruences. Indeed, the following are easily seen to be equivalent:

$$
\begin{aligned}
\mathscr{K} \models \mathscr{K} \text {-con } & {[\alpha \beta, \beta]=\alpha[\beta, \beta], } \\
\mathscr{K} \models_{\text {con }}[\alpha \beta, \beta]=0 & \rightarrow \alpha[\beta, \beta]=0, \\
\mathscr{K} \models_{\text {con }} \kappa(\alpha \beta, \beta)=0 & \rightarrow \alpha \cdot \kappa(\beta, \beta)=0, \\
\mathscr{K} \models_{\text {con }}[\alpha \beta, \beta]_{\mathscr{L}}=0 & \rightarrow \alpha[\beta, \beta]_{\mathscr{L}}=0 .
\end{aligned}
$$

In $(\mathrm{d}), \mathscr{L}$ can be any relatively modular quasivariety containing $\mathscr{K}$. Condition (c) is especially interesting. It shows that, for any algebra $\mathbf{A}$, there is an intrinsic condition that $\mathbf{A}$ must satisfy in order for $\mathbf{A}$ to belong to any relatively modular quasivariety which satisfies the relative commutator equation in (a).

Corollary 3.2. If $\mathscr{K}$ is a finitely generated, relatively modular quasivariety of groups, then $\mathscr{K}$ contains no non-Abelian nilpotent groups. Hence $\mathscr{K}$ is finitely axiomatizable. 
Proof. The hypotheses guarantee that $\mathscr{K}$ satisfies the above equivalent conditions (a)-(d). In condition (d) we may choose $\mathscr{L}$ to be the variety of groups. Then the $\mathscr{L}$-commutator is the usual commutator of group theory, so it follows that $\mathscr{K}$ contains no non-Abelian nilpotent groups. This establishes the first statement. The second statement follows from a result of $\mathrm{Ol}^{\prime}$ shanskii. In $[\mathrm{O}]$, one finds the result that the quasivariety generated by a finite group $\mathbf{G}$ is finitely axiomatizable iff all the Sylow subgroups of $\mathbf{G}$ are Abelian. (This condition is equivalent to the requirement that all nilpotent groups in the variety generated by $\mathbf{G}$ be Abelian.)

\section{Characterizations of Relatively Modular AND RELATIVELY DISTRIBUTIVE QUASIVARIETIES}

In $\S 2$, we developed a commutator theory for relatively modular quasivarieties. Unfortunately, very little is known about these quasivarieties. Several papers written in the past few years contain examples or results concerning the characterization of relatively distributive quasivarieties; for example, $[\mathrm{CzDz}$, D, DQS, Dz2, Dz3, K1, K2, P]. But none of these papers contains a complete characterization. We can find in the literature no example of a quasivariety which is claimed to be relatively modular that is not relatively distributive, or is not contained in a modular variety. In fact, only a very few known examples fail to be either a modular variety or a relatively distributive subquasivariety of a modular variety. The purpose of this section is to characterize relatively modular and relatively distributive quasivarieties. We will exhibit, in $\S 7$, natural examples of relatively modular quasivarieties which are not contained in any modular variety nor in any relatively distributive quasivariety.

Our first theorem may be viewed as a quasivariety analogue of Day's Theorem which characterizes congruence-modular varieties.

Theorem 4.1. A quasivariety $\mathscr{K}$ is relatively modular if and only if $\mathscr{K}$ satisfies the extension principle and the relative shifting lemma (Theorem 2.1(2)).

Proof. Theorems 1.1 and 2.1 already show that if $\mathscr{K}$ is relatively modular then the conditions listed must hold. We must show that if the conditions hold, then $\mathscr{K}$ is relatively modular. We will argue by contradiction.

Assume that $\mathscr{K}$ satisfies the extension principle, and possesses a finite set of pairs of terms $\Sigma_{s}$ fulfilling Theorem 2.1(2), but that $\mathscr{K}$ is not relatively modular. Then there is an $\mathbf{A} \in \mathscr{K}$ and $\alpha, \beta, \gamma \in \operatorname{Con}_{\mathscr{H}} \mathbf{A}$ such that $\alpha<\beta$, $\alpha \gamma=\beta \gamma$, and $\alpha+^{\prime} \gamma=\beta+^{\prime} \gamma$. Let $\delta=(\alpha+\gamma) \beta$. Of course, $\alpha \leq \delta \leq \beta$ so $\alpha \gamma=\delta \gamma$. By the extension principle, $\delta^{\prime}=\left(\alpha+^{\prime} \gamma\right) \beta=\beta$ so $\alpha<\delta$.

The definition of $\delta$ is such that $\alpha<\delta \leq \alpha+\gamma$, so there is an $n$ such that

$$
(\gamma \circ \alpha)^{n} \circ \gamma \cap \beta \nsubseteq \alpha \text {. }
$$

Let $k$ be the least $n$ with this property. $k$ is greater than 0 since $\gamma \beta=$ $\gamma \alpha \subseteq \alpha$. By the minimality of $k$,

$$
(\gamma \circ \alpha)^{k-1} \circ \gamma \cap \beta \subseteq \alpha,
$$

$$
(\gamma \circ \alpha)^{k} \cap \beta=(\gamma \circ \alpha)^{k-1} \circ \gamma \circ \alpha \cap \beta \subseteq \alpha .
$$


Choose a pair $(a, b) \in\left((\gamma \circ \alpha)^{k} \circ \gamma \cap \beta\right)-\alpha$ and a sequence of elements $a=$ $x_{0}, x_{1}, \ldots, x_{2 k+1}=b$ such that $\left(x_{i}, x_{i+1}\right)$ is a member of $\gamma$ when $i$ is even and a member of $\alpha$ if $i$ is odd. To reach a contradiction, we will consider two separate cases depending on whether $k$ is odd or even.

If $k$ is odd, then $k=2 r+1$ for some $r$. In this case, the "middle link" in the chain of elements connecting $a$ to $b,\left(x_{k}, x_{k+1}\right)$, is a member of $\alpha$. For any $\langle p, q\rangle \in \Sigma_{s}$ we have

$$
\begin{aligned}
& p^{\mathbf{A}}\left(a, x_{k}, x_{k+1}, b\right)(\gamma \circ \alpha)^{r} \circ \gamma p^{\mathbf{A}}(a, a, b, b) \\
& \quad=q^{\mathbf{A}}(a, a, b, b)(\gamma \circ \alpha)^{r} \circ \gamma q^{\mathbf{A}}\left(a, x_{k}, x_{k+1}, b\right) .
\end{aligned}
$$

Further,

$$
\begin{aligned}
& p^{\mathbf{A}}\left(a, x_{k}, x_{k+1}, b\right) \beta p^{\mathbf{A}}\left(a, x_{k}, x_{k}, a\right) \\
& \quad=q^{\mathbf{A}}\left(a, x_{k}, x_{k}, a\right) \beta q^{\mathbf{A}}\left(a, x_{k}, x_{k+1}, b\right) .
\end{aligned}
$$

Now, $(\gamma \circ \alpha)^{r} \circ \gamma \circ(\gamma \circ \alpha)^{r} \circ \gamma=(\gamma \circ \alpha)^{2 r} \circ \gamma \subseteq(\gamma \circ \alpha)^{k}$ and $(\gamma \circ \alpha)^{k} \cap \beta \subseteq \dot{\alpha}$. Hence, $p^{\mathbf{A}}\left(a, x_{k}, x_{k+1}, b\right) \alpha q^{\mathbf{A}}\left(a, x_{k}, x_{k+1}, b\right)$ holds. The pair $\langle p, q\rangle$ was arbitrary and $\alpha$ is a $\mathscr{K}$-congruence, so the quasi-equation in Theorem 2.1(2)(ii) implies that $(a, b) \in \alpha$.

Now assume that $k$ is positive and even and that $k=2 r$. Then $\left(x_{k-1}, x_{k}\right)$ is a member of $\alpha$. As before,

$$
\begin{aligned}
& p^{\mathbf{A}}\left(a, x_{k-1}, x_{k}, b\right)(\gamma \circ \alpha)^{r} p^{\mathbf{A}}\left(a, a, x_{2 k-1}, x_{2 k-1}\right) \\
& \quad=q^{\mathbf{A}}\left(a, a, x_{2 k-1}, x_{2 k-1}\right) \alpha \circ(\gamma \circ \alpha)^{r} q^{\mathbf{A}}\left(a, x_{k-1}, x_{k}, b\right),
\end{aligned}
$$

so $p^{\mathbf{A}}\left(a, x_{k-1}, x_{k}, b\right) \quad(\gamma \circ \alpha)^{2 r} \quad q^{\mathbf{A}}\left(a, x_{k-1}, x_{k}, b\right)$. We also have that $p^{\mathbf{A}}\left(a, x_{k-1}, x_{k}, b\right) \quad \beta \quad q^{\mathbf{A}}\left(a, x_{k-1}, x_{k}, b\right)$ holds by the same argument that we used above. Since

$$
(\gamma \circ \alpha)^{2 r} \cap \beta=(\gamma \circ \alpha)^{k} \cap \beta \subseteq \alpha,
$$

we again obtain that $p^{\mathbf{A}}\left(a, x_{k-1}, x_{k}, b\right) \alpha q^{\mathbf{A}}\left(a, x_{k-1}, x_{k}, b\right)$ holds. Since $\left(x_{k-1}, x_{k}\right) \in \alpha$, this provides the same contradiction that we reached in the last paragraph.

The two cases we have argued are exhaustive, so the proof is finished.

It is worth pointing out exactly where the extension principle was used in the proof of Theorem 4.1. The only time it was used was in the second paragraph of the proof where we proved that $\alpha<\delta$ by observing that $\alpha \leq \delta$ and $\alpha^{\prime}=\alpha<$ $\beta=\delta^{\prime}$. We could have proved this with a condition weaker than the extension principle:

$$
\mathscr{K} \models_{\text {con }} \psi^{\prime} \theta=0 \rightarrow \psi^{\prime} \theta^{\prime}=0 .
$$

To see this, let $\theta=(\alpha+\gamma) / \alpha \in \operatorname{Con} \mathbf{A} / \alpha$ and let $\psi=\psi^{\prime}=\beta / \alpha \in \operatorname{Con} \mathbf{A} / \alpha$. Then $\psi^{\prime} \theta=(\beta(\alpha+\gamma)) / \alpha=\delta / \alpha$. If $\alpha=\delta$ then, since $\mathbf{A} / \alpha \in \mathscr{H}$ and $\mathscr{K} \vDash$ con $\psi^{\prime} \theta=0 \rightarrow \psi^{\prime} \theta^{\prime}=0$, we get that $0=\psi^{\prime} \theta^{\prime}=\beta / \alpha$. This is false since $\alpha<\beta$; hence $\alpha<\delta$. Thus, in the presence of the terms given by the relative shifting lemma, the extension principle is equivalent to the condition that $\mathscr{K} \models_{\text {con }} \psi^{\prime} \theta=0 \rightarrow \psi^{\prime} \theta^{\prime}=0$.

We will call the terms involved in the set $\Sigma_{s}$, which fulfill Theorem 2.1(2) for $\mathscr{K}$, quasi-Day terms for $\mathscr{K}$. More generally, we will call a condition postulating 
the existence of a finite set of terms satisfying certain quasi-equations a quasi$\mathrm{Mal}^{\prime} \mathrm{cev}$ condition. The existence of a congruence-modular variety with a subquasivariety that fails to be relatively modular (Example 1.3) demonstrates the impossibility of characterizing relative modularity by any set of quasi-Mal'cev conditions. Nevertheless, the previous theorem shows that relative modularity is the join of a quasi-Mal'cev condition and the extension principle. If $\mathscr{K}$ is a congruence-modular variety with Day terms $m_{0}(x, y, z, u), \ldots$, $m_{n}(x, y, z, u)$ satisfying the usual equations, then we may choose $\Sigma_{s}$ to be the set of pairs $\left\{\left\langle m_{i}(x, y, z, u), m_{i+1}(x, y, z, u)\right\rangle: i<n, i\right.$ even $\}$. It is an easy exercise to check that this (finite) set of pairs of terms satisfies the relative shifting lemma for $\mathscr{K}$.

It is also easy to show that a quasivariety satisfies the extension principle iff its finitely generated algebras do. Further, the proof of Theorem 2.1 shows that a quasivariety has quasi-Day terms if its 4-generated free algebra has a relatively modular congruence lattice. Together, these facts imply that a locally finite quasivariety is relatively modular iff its finite algebras have modular relative congruence lattices.

The next theorem is an easily stated (and easily proven) characterization of relatively distributive quasivarieties.

Theorem 4.2. A quasivariety $\mathscr{K}$ is relatively distributive iff for every $\mathbf{A} \in \mathscr{K}$, every completely meet-irreducible member of $\mathbf{C o n}_{\mathscr{K}} \mathbf{A}$ is a meet-prime member of Con A.

Proof. The necessity follows easily from the extension principle; the sufficiency is also a quite straightforward argument.

The next theorem combines quasivariety analogues of Jónsson's Theorem characterizing congruence-distributive varieties with analogues of the com mutator-theoretic characterization of congruence-distributive varieties found in [FM].

Theorem 4.3. The following conditions are equivalent for a quasivariety $\mathscr{K}$ :

(1) $\mathscr{K}$ is relatively distributive.

(2) $\mathscr{K}$ is relatively modular and satisfies the commutator equation $[\alpha, \beta]=$ $\alpha \cdot \beta^{\prime}$.

(3) $\mathscr{K}$ is relatively modular and satisfies $[\alpha, \alpha]=0 \rightarrow \alpha=0$.

(4) $\mathscr{K}$ satisfies the extension principle, and there exist a finite set $\Sigma_{d}$ of triples of terms $\langle r(x, y, z), s(x, y, z), t(x, y, z)\rangle$ in the language of $\mathscr{K}$ such that: (i) if $\langle r, s, t\rangle \in \Sigma_{d}$ then the equations

$$
\begin{gathered}
r(x, y, x) \approx s(x, y, x) \approx t(x, y, x), \\
r(x, x, y) \approx s(x, x, y), \quad s(x, y, y) \approx t(x, y, y)
\end{gathered}
$$

are valid in $\mathscr{K}$; (ii) the quasi-equation

$$
\begin{aligned}
& \bigwedge_{\langle r, s, t\rangle \in \Sigma_{d}}(r(x, y, z) \approx s(x, y, z) \& s(x, y, z) \approx t(x, y, z)) \rightarrow x \approx z \\
& \quad \text { is valid in } \mathscr{K} .
\end{aligned}
$$

(5) $\mathscr{K}$ satisfies the extension principle, and there exist a finite set $\Sigma_{d}^{\prime}$ of quadruples of terms $\left\langle r(x, y, z), s(x, y, z), s^{\prime}(x, y, z), t(x, y, z)\right\rangle$ in the language of $\mathscr{K}$ such that: (i) If $\left\langle r, s, s^{\prime}, t\right\rangle \in \Sigma_{d}^{\prime}$ then the equations

$$
r(x, y, x) \approx s(x, y, x), \quad s^{\prime}(x, y, x) \approx t(x, y, x),
$$




$$
r(x, x, y) \approx s(x, x, y), \quad s^{\prime}(x, y, y) \approx t(x, y, y)
$$

are valid in $\mathscr{R}$; (ii) the quasi-equation

$$
\bigwedge_{\left\langle r, s, s^{\prime}, t\right\rangle \in \Sigma_{d}^{\prime}}\left(r(x, y, z) \approx s(x, y, z) \& s^{\prime}(x, y, z) \approx t(x, y, z)\right) \rightarrow x \approx z
$$

is valid in $\mathscr{K}$.

Proof. It was shown that (1) implies (2) in Example 2.7. Conversely, the additivity of the commutator (property (2) in Theorem 2.13) shows that (2) implies (1). The proofs that (2) implies (3) and that (4) implies (5) are obvious. To show that (3) implies (1), we will argue by contradiction. If (3) holds but (1) fails, then $\mathscr{K}$ is relatively modular, but not relatively distributive. There must be an algebra $\mathbf{A} \in \mathscr{K}$ with distinct congruences $\alpha, \beta, \gamma \in \operatorname{Con}_{\mathscr{K}} \mathbf{A}$ such that $\alpha \beta=\alpha \gamma=\beta \gamma$ and $\alpha+^{\prime} \beta=\alpha+^{\prime} \gamma=\beta+^{\prime} \gamma=\delta$. Necessarily, $\alpha<\delta$. This means that

$$
[\delta, \delta]=\left[\alpha+^{\prime} \beta, \alpha+^{\prime} \gamma\right] \leq \alpha+^{\prime}[\beta, \gamma] \leq \alpha .
$$

Hence, in $\mathbf{B}=\mathbf{A} / \alpha$ we have a congruence $\theta=\delta / \alpha$ for which $[\theta, \theta]=0$ even though $\theta \neq 0$. This is a contradiction. This shows that the first three conditions are equivalent.

The argument that produces the terms in (4) is very similar to the argument we used in Theorem 2.1. To show that (1) implies (4), let $\mathbf{F}=\mathbf{F}_{\mathscr{K}}(x, y, z)$ be a 3-generated free algebra in $\mathscr{K}$. Consider the $\mathscr{K}$-congruences $\alpha, \beta$, and $\gamma$ generated by $\{(x, z)\},\{(x, y)\}$, and $\{(y, z)\}$, respectively. If $\operatorname{tr}(X)$ denotes the transitive closure of the relation $X \subseteq F^{2}$ then, since $\mathscr{K}$ is relatively distributive,

$$
(x, z) \in \alpha\left(\beta+^{\prime} \gamma\right)=\alpha \beta+^{\prime} \alpha \gamma=[\operatorname{tr}(\alpha \beta \circ \alpha \gamma)]^{\prime} .
$$

Hence, there is a finite subset $T$ of $\alpha \beta \circ \alpha \gamma$ such that $[\operatorname{tr}(T)]^{\prime}$ contains $(x, z)$. For each $\left\langle r^{\mathbf{F}}, t^{\mathbf{F}}\right\rangle \in T$, where $r$ and $t$ are terms, there is a ternary term $s(x, y, z)$ such that $r^{\mathbf{F}} \alpha \beta s^{\mathbf{F}} \alpha \gamma t^{\mathbf{F}}$. If we let $\Sigma_{d}$ be a set of such triples of terms $\left\{\langle r, s, t\rangle:\left\langle r^{\mathbf{F}}, t^{\mathbf{F}}\right\rangle \in T\right\}$, then it is straightforward to verify that the quasi-equations listed in (4) hold. Of course, (1) implies that $\mathscr{K}$ satisfies the extension principle by Theorem 1.1. This finishes the proof that (1) implies (4).

We can finish the proof by showing that (5) implies (3). Assume that (5) holds. For each $\left\langle r, s, s^{\prime}, t\right\rangle \in \Sigma_{d}^{\prime}$ we define two pairs $\left\langle p_{1}, q_{1}\right\rangle$ and $\left\langle p_{2}, q_{2}\right\rangle$ by

$$
\begin{array}{ll}
p_{1}(x, y, z, u)=r(x, y, u), & q_{1}(x, y, z, u)=s(x, y, u), \\
p_{2}(x, y, z, u)=s^{\prime}(x, z, u), & q_{2}(x, y, z, u)=t(x, z, u) .
\end{array}
$$

If we let $\Sigma_{s}$ be the (finite) collection of all pairs of the form $\left\langle p_{1}, q_{1}\right\rangle$ and $\left\langle p_{2}, q_{2}\right\rangle$ that arise this way, one finds that this set satisfies Theorem 2.1(2) for $\mathscr{K}$. Hence, condition (5) implies that $\mathscr{K}$ satisfies the extension principle and has quasi-Day terms. By Theorem $4.1, \mathscr{K}$ is relatively modular. Now suppose that $\mathbf{A} \in \mathscr{K}$ and $\alpha \in$ Con $\mathbf{A}$ satisfies $[\alpha, \alpha]=0$. In other words, suppose that $Z(\alpha, \alpha)$ holds. Choose $(a, b) \in \alpha$ and assume for the moment that $a \neq b$. It is not true that $r^{\mathbf{A}}(a, b, b)=s^{\mathbf{A}}(a, b, b)$ and $s^{\prime \mathbf{A}}(a, b, b)=t^{\mathbf{A}}(a, b, b)$ for all $\left\langle r, s, s^{\prime}, t\right\rangle \in \Sigma_{d}^{\prime}$ by $5\left(\right.$ ii), but at least ${s^{\prime}}^{\mathbf{A}}(a, b, b)=t^{\mathbf{A}}(a, b, b)$ does hold. Hence, we can find a tuple in $\Sigma_{d}^{\prime}$ for which $r^{\mathbf{A}}(a, b, b) \neq s^{\mathbf{A}}(a, b, b)$. 
Let $f(x, y)=r^{\mathbf{A}}(x, b, y)$ and let $g(x, y)=s^{\mathbf{A}}(x, b, y)$. The equations listed in (5) imply that $f(a, a)=g(a, a), f(b, a)=g(b, a)$, and $f(b, b)$ $=g(b, b)$, but we have chosen these polynomials so that $f(a, b) \neq g(a, b)$. This contradicts the fact that $Z(\alpha, \alpha)$ holds unless our assumption that $a \neq b$ is false. Since $(a, b) \in \alpha$ was arbitrary, $\alpha=0$. This shows that (3) holds, finishing the proof of Theorem 4.3.

(After the first draft of this paper was written we learned that A. M. Nurakunov had discovered a proof that a quasivariety $\mathscr{K}$ is relatively distributive iff $\mathscr{K}_{\text {RFSI }} \subseteq \mathscr{K}_{\text {FSI }}$ and $\mathscr{K}$ satisfies quasi-equations listed in Theorem 4.3(5). The condition $\mathscr{K}_{\text {RFSI }} \subseteq \mathscr{K}_{\text {FSI }}$, that every relatively finitely subdirectly irreducible member of $\mathscr{K}$ is finitely subdirectly irreducible, is weaker than the extension principle, so Nurakunov's result is a better characterization of relatively distributive quasivarieties than condition (5) of Theorem 4.3. Condition (4) of Theorem 4.3 can be improved similarly. In [Dz3], W. Dziobiak proved Nurakunov's result for subquasivarieties of a congruence permutable variety.)

We will call the terms in condition (4) quasi-Jónsson terms.

The next few results concern locally finite quasivarieties. We will use the terminology and methods of tame congruence theory. The reader not familiar with the theory is referred to [HM].

Lemma 4.4. Assume that $\mathbf{A} \in \mathscr{K}$ is finite and that $\alpha \in \mathrm{Con} \mathbf{A}$ is a minimal nonzero congruence. If $\mathscr{K}$ is relatively modular, then $\operatorname{typ}(0, \alpha) \in\{\mathbf{2}, \mathbf{3}, \mathbf{4}\}$. If $\mathscr{K}$ is relatively distributive, then $\operatorname{typ}(0, \alpha) \in\{\mathbf{3}, \mathbf{4}\}$.

Proof. Assume first that $\mathscr{K}$ is relatively modular. Let $\Sigma_{s}$ be a set of pairs of terms satisfying the shifting lemma for $\mathscr{K}$. We need to eliminate the possibility that $\operatorname{typ}(0, \alpha) \in\{\mathbf{1}, \mathbf{5}\}$. Choose $U \in \mathbf{M}_{\mathbf{A}}(0, \alpha)$ and let $T$ be a $(0, \alpha)$-trace in $U$. Pick distinct elements $a, b \in T$. Since $a \neq b$, there is a pair $\langle p, q\rangle \in \Sigma_{s}$ such that $p^{\mathbf{A}}(a, b, b, b) \neq q^{\mathbf{A}}(a, b, b, b)$. However,

$$
p^{\mathbf{A}}(a, b, b, b) \alpha p^{\mathbf{A}}(b, b, b, b)=q^{\mathbf{A}}(b, b, b, b) \alpha q^{\mathbf{A}}(a, b, b, b),
$$

so $p^{\mathbf{A}}(a, b, b, b)\left(\alpha-0_{\mathbf{A}}\right) q^{\mathbf{A}}(a, b, b, b)$. There is a unary polynomial of $\mathbf{A}, f$, such that $f(A)=U$ and $f p^{\mathbf{A}}(a, b, b, b)\left(\alpha-0_{\mathbf{A}}\right) f q^{\mathbf{A}}(a, b, b, b)$. By suitably altering $f$ if necessary, we may assume that $f p^{\mathbf{A}}(a, b, b, b)$ and $f q^{\mathbf{A}}(a, b, b, b)$ are distinct elements of $T$. Now $U$ is closed under $f p^{\mathbf{A}}$ and under $f q^{\mathbf{A}}$, and $T$ is an $\left.\alpha\right|_{U}$-class of $U$; so $T$ is closed under $f p^{\mathbf{A}}$ and under $f q^{\mathbf{A}}$. We are now in a position to show that $\operatorname{typ}(0, \alpha) \notin\{\mathbf{1}, \mathbf{5}\}$.

For our first case, assume that $\operatorname{typ}(0, \alpha)=\mathbf{1}$. Then $\mathbf{A I}_{T}$ is essentially unary, so $f p^{\mathbf{A}}(x, y, z, u)$ and $f q^{\mathbf{A}}(x, y, z, u)$, restricted to $T$, depend on at most one variable. But $f p^{\mathbf{A}}(a, b, b, b) \neq f q^{\mathbf{A}}(a, b, b, b)$ and $f p^{\mathbf{A}}(b, b, b, b)$ $=f q^{\mathbf{A}}(b, b, b, b)$, so at least one of these polynomials depends on the first variable. By a similar argument, $f p^{\mathbf{A}}(a, a, b, b)=f q^{\mathbf{A}}(a, a, b, b)$ shows that one of them depends on the second variable and $f p^{\mathbf{A}}(a, b, b, a)=$ $f q^{\mathbf{A}}(a, b, b, a)$ shows that one of them depends on the last variable. This forces one of them to depend on at least two variables (when restricted to $T$ ), which is impossible. Hence, $\operatorname{typ}(0, \alpha)$ is not 1 .

Now assume that $\operatorname{typ}(0, \alpha)=\mathbf{5}$. Then $|T|=2$ and $\mathbf{A I}_{T}$ is polynomially equivalent to a semilattice. Since $a$ and $b$ were chosen arbitrarily, we may assume that $b$ is the label that we gave to the element of $T$ that is the absorbing element for all polynomials. Since $f p^{\mathbf{A}}(a, b, b, b)$ and $f q^{\mathbf{A}}(a, b, b, b)$ are 
different, we may assume that $f p^{\mathbf{A}}(a, b, b, b)=a$ and $f q^{\mathbf{A}}(a, b, b, b)=$ $b$. This forces the conclusion that $f p^{\mathbf{A}}$, restricted to $T$, does not depend on its last three variables. Hence, $f q^{\mathbf{A}}(a, a, b, b)=f p^{\mathbf{A}}(a, a, b, b)=a$, which implies that $f q^{\mathbf{A}}$, restricted to $T$, does not depend on its last two variables. This gives us the contradiction that

$$
\begin{aligned}
a & =f p^{\mathbf{A}}(a, b, b, b)=f p^{\mathbf{A}}(a, b, b, a) \\
& =f q^{\mathbf{A}}(a, b, b, a)=f q^{\mathbf{A}}(a, b, b, b)=b .
\end{aligned}
$$

Thus $\operatorname{typ}(0, \alpha)$ is not 5 .

To finish the proof we need to show that under the stronger assumption that $\mathscr{K}$ is relatively distributive, we can rule out the possibility that $\operatorname{typ}(0, \alpha)$ is 2 . With an argument similar to that in the first paragraph, using quasi-Jónsson terms instead of quasi-Day terms, we can arrange it so that $r^{\mathbf{A}}(a, b, b)\left(\alpha-0_{\mathbf{A}}\right) s^{\mathbf{A}}(a, b, b)$, where $r$ and $s$ are components of a triple $\langle r, s, t\rangle \in \Sigma_{d}$. There is a unary polynomial of $\mathbf{A}, g$, such that $\operatorname{gr}^{\mathbf{A}}(a, b, b)$ and $g s^{\mathbf{A}}(a, b, b)$ are distinct elements of $T$. Further, $T$ is closed under $g r^{\mathbf{A}}$ and $g s^{\mathbf{A}}$. If we assume that $\operatorname{typ}(0, \alpha)=\mathbf{2}$, the algebra $\mathbf{A I}_{T}$ is polynomially equivalent to a module. Choosing $b$ as zero element for this module, set

$$
h(x, y, z)=g r^{\mathbf{A}}(x, y, z)-g s^{\mathbf{A}}(x, y, z) .
$$

One can quickly verify that $h(x, y, x)=h(x, x, y)=b$ for all $x, y \in T$, but that $h(a, b, b) \neq b$. That is, $h(a, a, a)=h(a, a, b)$, but $h(a, b, a)=$ $b \neq h(a, b, b)$. This is a failure of the (one-) term condition in $\mathbf{A I}_{T}$, which is impossible. Hence, $\operatorname{typ}(0, \alpha)$ is not 2 . This finishes the proof.

The next theorem is weaker than Theorem 4.1 in that it is not a complete characterization of relative modularity for locally finite quasivarieties. Nevertheless, it may be easier to apply than Theorem 4.1. The monolith of a subdirectly irreducible algebra is the smallest nonzero congruence of the algebra.

Theorem 4.5. Let $\mathscr{K}$ be a locally finite quasivariety. The following conditions imply that $\mathscr{K}$ is relatively modular:

(1) $\mathscr{K}$ satisfies the extension principle.

(2) Every finite $\mathbf{A} \in \mathscr{K}_{\mathrm{SI}}$ has monolith of type 2, 3, or $\mathbf{4}$.

(3) Where $\mu_{\mathbf{A}}$ is the monolith of $\mathbf{A}$, each $U \in \mathbf{M}_{\mathbf{A}}\left(0, \mu_{\mathbf{A}}\right)$ has empty tail. Conversely, if $\mathscr{K}$ is relatively modular, then (1) and (2) must hold.

Proof. The converse is by Theorem 1.1 and Lemma 4.4. We need to prove the forward direction.

Assume that (1), (2), and (3) hold for $\mathscr{K}$. We will assume that $\mathscr{K}$ is not relatively modular and argue to a contradiction. Since $\mathscr{K}$ is locally finite, we can find a finite algebra $\mathbf{A} \in \mathscr{K}$ which has a nonmodular relative congruence lattice. Say that $\alpha, \beta, \gamma \in \operatorname{Con}_{\not \prime} \mathbf{A}$ and that $\alpha<\beta, \alpha \gamma=\beta \gamma$, and $\alpha+^{\prime} \gamma$ $=\beta+^{\prime} \gamma$. Set $\delta=(\alpha+\gamma) \beta$. By $(1), \delta^{\prime}=\beta$ so $\alpha<\delta$. There must exist a congruence $\psi \in \operatorname{Con}_{\mathscr{H}} \mathbf{A}$ which is completely meet-irreducible in $\mathbf{C o n}_{\not{H}} \mathbf{A}$ such that $\alpha \leq \psi$ and $\delta \notin \psi \psi$. By condition (1) and the fact that $\mathbf{A}$ is finite, $\psi$ is completely meet-irreducible in $\mathbf{C o n} \mathbf{A}$ as well. Let $\psi^{*}$ denote the unique upper cover of $\psi$. Now, pick $U \in \mathbf{M}_{\mathbf{A}}\left(\psi, \psi^{*}\right)$. Since the restriction map is a lattice homomorphism from $\operatorname{Con} \mathbf{A}$ to $\operatorname{Con} \mathbf{A I}_{L^{\prime}}$, we have

$$
\left.\alpha\right|_{U} \leq\left.\psi\right|_{U}<\left.\psi^{*}\right|_{U} \leq\left.(\delta+\psi)\right|_{U}=\left.\delta\right|_{U}+\left.\psi\right|_{U}
$$


Hence, $\left.\alpha\right|_{U}<\left.\delta\right|_{U}$. Since $\alpha \gamma=\delta \gamma$ and $\alpha+\gamma=\delta+\gamma$, we also have $\left.\left.\alpha\right|_{U} \gamma\right|_{U}=$ $\left.\left.\delta\right|_{U} \gamma\right|_{U}$ and $\left.\alpha\right|_{U}+\left.\gamma\right|_{U}=\left.\delta\right|_{U}+\left.\gamma\right|_{U}$. This shows that $\operatorname{Con} \mathbf{A I}_{U}$ is nonmodular. However, conditions (2) and (3) imply that $\mathbf{A I}_{U}$ is $\left\langle\left.\psi\right|_{U},\left.\psi^{*}\right|_{U}\right\rangle$-minimal of type 2, 3, or $\mathbf{4}$ and that $\mathbf{A I _ { U }}$ has no tail, therefore $\mathbf{A I}_{U}$ is $\mathrm{E}$-minimal. This implies that either $U$ has only two elements or that $\mathbf{A I}_{U}$ is $\mathrm{Mal}^{\prime}$ cev. In either case, $\mathbf{A I}_{U}$ has permuting congruences which implies that $\mathbf{C o n} \mathbf{A I}_{U}$ is modular. This is the contradiction that we sought.

An analogous but stronger result is true for relatively distributive quasivarieties.

Theorem 4.6. Let $\mathscr{K}$ be a locally finite quasivariety. The following conditions imply that $\mathscr{K}$ is relatively distributive:

(1) The finite algebras in $\mathscr{K}_{\mathrm{RSI}}$ are subdirectly irreducible.

(2) Every finite $\mathbf{A} \in \mathscr{K}_{\mathrm{SI}}$ has monolith of type $\mathbf{3}$ or $\mathbf{4}$.

(3) Where $\mu_{\mathbf{A}}$ is the monolith, each $U \in \mathbf{M}_{\mathbf{A}}\left(0, \mu_{\mathbf{A}}\right)$ has empty tail. Conversely, if $\mathscr{K}$ is relatively distributive, then (1) and (2) must hold.

Proof. The extension principle implies that the zero congruence of a relatively subdirectly irreducible algebra is at least meet-irreducible as an ordinary congruence; for a finite algebra it must be even completely meet-irreducible. Hence, condition (1) is implied by the extension principle. If $\mathscr{K}$ is relatively distributive then (1) must hold. Further, Lemma 4.4 shows that (2) holds, in this case. This proves the converse.

To prove the sufficiency of the conditions, we are going to argue by contradiction again. Suppose that (1), (2), and (3) hold and that $\mathscr{K}$ fails to be relatively distributive. The remarks that we made after Theorem 4.1 , which apply equally well to Theorem 4.3, show that we can find a finite algebra $\mathbf{A} \in \mathscr{K}$ which fails to have a distributive relative congruence lattice. Say that $\alpha, \beta, \gamma \in \operatorname{Con}_{\mathscr{H}} \mathbf{A}$ and

$$
\theta=\alpha\left(\beta+^{\prime} \gamma\right)>\alpha \beta+^{\prime} \alpha \gamma=\delta .
$$

There is a congruence $\psi$ which is completely meet-irreducible in $\operatorname{Con}_{\mathscr{K}} \mathbf{A}$ such that $\delta \leq \psi$ and $\theta \leq \psi$. Condition (1) guarantees that $\psi$ is completely meetirreducible as an ordinary congruence. Let $\psi^{*}$ denote the unique upper cover of $\psi$. Now, if $\beta \leq \psi$ and $\gamma \leq \psi$ then $\theta \leq \beta+^{\prime} \gamma \leq \psi$, which is false. Therefore, we may assume that $\beta \leq \psi$. Further, $\alpha \notin \psi$. Now pick $U \in \mathbf{M}_{\mathbf{A}}\left(\psi, \psi^{*}\right)$. Conditions (2) and (3) imply that $|U|=2$. Hence, $\mathbf{A I}_{U}$ is an algebra with exactly two congruences: $0_{U}$ and $1_{U}$. Since $\left.\psi\right|_{U}<\left.\psi^{*}\right|_{U}$, it must be that $\left.\psi\right|_{U}$ $=0_{U}$ and $\left.\psi^{*}\right|_{U}=1_{U}$. Restriction of congruences is a lattice homomorphism from Con $\mathbf{A}$ to Con $\mathbf{A} \mathbf{I}_{U}$ and this homomorphism could only be the map

$$
\xi \mapsto \begin{cases}0_{U} & \text { if } \xi \leq \psi, \\ 1_{U} & \text { otherwise. }\end{cases}
$$

Hence, $\left.\alpha\right|_{U}=1_{U}$ and $\left.\beta\right|_{U}=11_{U}$ and this should force $\left.(\alpha \beta)\right|_{U}=\left(\left.\alpha\right|_{U}\right)\left(\left.\beta\right|_{U}\right)$ $=1_{U}$. But $\alpha \beta \leq \psi$, so $\left.(\alpha \beta)\right|_{U}=0_{U}$. This contradiction finishes the proof.

If $\mathscr{K}$ is generated by a finite set $M$ of finite algebras, then $\mathscr{K}_{\mathrm{RSI}} \subseteq \boldsymbol{S}(M)$. This allows us to rewrite condition (1) in Theorem 4.6, for finitely generated quasivarieties, as $\mathscr{K}_{\mathrm{RSI}} \subseteq \mathscr{K}_{\mathrm{SI}}$. As we mentioned, the hypothesis that $\mathscr{K}_{\mathrm{RSI}} \subseteq$ $\mathscr{K}_{\mathrm{SI}}$ is much weaker than the extension principle. Indeed, showing that every 
relatively subdirectly irreducible algebra in a finitely generated quasivariety $\mathscr{K}$ is subdirectly irreducible can be accomplished by examining the algebras in $\boldsymbol{S}(\boldsymbol{M})$ and the homomorphisms between them. One may now ask if Theorem 4.5 remains valid if we weaken its condition (1) to the condition $\mathscr{K}_{\mathrm{RSI}} \subseteq \mathscr{K}_{\mathrm{SI}}$. The answer is no. If we let $\mathbf{H}$ be the quaternion group and $\mathscr{K}=\boldsymbol{S P}(\mathbf{H})$, then $\mathscr{K}_{\text {RSI }} \subseteq \mathscr{K}_{\text {SI }}$ and conditions (2) and (3) of Theorem 4.5 hold. However, $\mathscr{K}$ is not relatively modular. (That this is so can be deduced from Theorem 3.1 or Theorem 4.8(3)(ii).)

One of the appealing aspects of Theorem 4.6 is that it allows us to prove that a finite set of finite algebras generates a relatively distributive quasivariety just by checking that the generating algebras satisfy certain conditions. The other theorems that we have given require us to verify that the generated quasivariety satisfies the extension principle; and we do not know a general test to check that the extension principle holds. However, this problem can be avoided if one knows merely that the generated quasivariety is contained in some relatively modular quasivariety. This will become apparent after we characterize the relatively modular subquasivarieties of a given relatively modular quasivariety.

In characterizing relative modularity for the subquasivarieties of a given relatively modular quasivariety, we will require an extension of Ralph Freese's notion of similarity of two subdirectly irreducible algebras in a congruencemodular variety. Suppose that $\mathscr{V}$ is a congruence-modular variety. Two subdirectly irreducible algebras $\mathbf{A}$ and $\mathbf{B}$ in $\mathscr{V}$ are said to be similar in $\mathscr{V}$ iff there is an algebra $\mathbf{C} \in \mathscr{V}$ and congruences $\alpha, \alpha^{\star}, \beta, \beta^{\star}, \delta, \gamma$ of $\mathbf{C}$ such that $\mathbf{C} / \alpha \cong \mathbf{A}, \mathbf{C} / \beta \cong \mathbf{B}, \alpha^{\star}$ and $\beta^{\star}$ are the unique covers of $\alpha$ and $\beta$, and $\alpha^{\star} / \alpha \searrow \gamma / \delta \nearrow \beta^{\star} / \beta$. (By $\alpha^{\star} / \alpha \searrow \gamma / \delta$, we mean that $\alpha^{\star}=\alpha+\gamma$ and $\delta$ $=\alpha \gamma$. Similarly, $\gamma / \delta \nearrow \beta^{\star} / \beta$ means that $\beta^{\star}=\gamma+\beta$ and $\delta=\gamma \beta$.) The relation of similarity is an equivalence relation on the class of subdirectly irreducible algebras in $\mathscr{V}$; when $\mathbf{A}, \mathbf{B}, \mathbf{C}$ are related as above, we can always take $\delta=\alpha \beta$, and thus we can always arrange that $\alpha \beta=0_{C}$ and $\mathbf{C}$ is a subdirect product of $\mathbf{A}$ and $\mathbf{B}$.

Now if $\mathscr{K}$ is a quasivariety, then we say that finitely subdirectly irreducible algebras $\mathbf{A}$ and $\mathbf{B}$ are similar in $\mathscr{K}$ iff they are members of $\mathscr{K}$ and there exist $\mathbf{C} \in \mathscr{K}$ and $\mathscr{K}$-congruences $\alpha, \beta, \alpha^{\star}, \beta^{\star}, \delta, \gamma$ of $\mathbf{C}$ such that $\mathbf{C} / \alpha \cong \mathbf{A}$, $\mathbf{C} / \beta \cong \mathbf{B}, \alpha<\alpha^{\star}$ and $\beta<\beta^{\star}$; and $\alpha^{\star} / \alpha \searrow \gamma / \delta \nearrow \beta^{\star} / \beta$ in the lattice $\operatorname{Con}_{\mathscr{K}} \mathbf{C}$. We will write this as $\mathbf{A} \sim_{\mathscr{K}} \mathbf{B}$.

Suppose that $\mathscr{L} \subseteq \mathscr{K}$, where $\mathscr{K}$ and $\mathscr{L}$ are quasivarieties and $\mathscr{K}$ is relatively modular. We say that $\mathscr{L}_{F S I}$ is closed under similarity in $\mathscr{K}$ if whenever we have finitely subdirectly irreducible algebras $\mathbf{A}$ and $\mathbf{B}$ such that $\mathbf{A} \sim_{\mathscr{K}} \mathbf{B}$, $\mathbf{A} \in \mathscr{L}$, and $\mathbf{B} \in \boldsymbol{H}(\mathscr{L})$, then we necessarily have $\mathbf{B} \in \mathscr{L}$. Note that, as in the definition of similarity for subdirectly irreducible algebras in a modular variety, we can always take $\mathbf{C}$ (in the definition of similar algebras) to be a subdirect product of $\mathbf{A}$ and $\mathbf{B}$ (so long as $\mathscr{K}$ is relatively modular). Note also that if $\mathbf{A}, \mathbf{B} \in \mathscr{L}$ and both $\mathscr{K}$ and $\mathscr{L}$ are relatively modular (and $\mathscr{L} \subseteq \mathscr{K}$ ) then $\mathbf{A} \sim \mathscr{K} \mathbf{B}$ is equivalent to $\mathbf{A} \sim \mathscr{L} \mathbf{B}$. Observe, finally, that if $\mathbf{A}$ and $\mathbf{B}$ are finitely subdirectly irreducible algebras and are similar in $\mathscr{K}$, then $\mathbf{A}$ is relatively subdirectly irreducible in $\mathscr{K}$ iff $\mathbf{B}$ is, and if they are subdirectly irreducible and $\mathscr{K}$ is a variety, then they are similar in the sense of Freese's definition.

Now assume that $\mathbf{A} \sim_{\mathscr{K}} \mathbf{B}$, where $\mathscr{K}$ is a relatively modular quasivariety. 
Assuming also that $\mathbf{A} \neq \mathbf{B}$, we can show that $\mathbf{A}$ (as well as $\mathbf{B}$ ) has a nonzero Abelian congruence. Thus if $\mathscr{K}$ is relatively distributive then the relation of $\mathscr{K}$-similarity is not very interesting-it is just the same as isomorphism. To see this, assume that $\mathbf{A} \neq \mathbf{B}$. Using the notation of the last paragraph, it follows that $\alpha \neq \beta$, and hence that $\beta \not \alpha$, since $\alpha \gamma=\beta \gamma$ and $\operatorname{Con}_{\mathscr{K}} \mathbf{C}$ is modular. Hence $\mu>\alpha$, where $\mu=\left(\alpha+^{\prime} \beta\right)\left(\alpha+^{\prime} \gamma\right)$. The argument concludes with the calculation

$$
[\mu, \mu] \leq\left[\alpha+^{\prime} \beta, \alpha+^{\prime} \gamma\right] \leq \alpha+^{\prime}[\beta, \gamma] \leq \alpha+^{\prime} \beta \gamma=\alpha,
$$

showing that $[\mu, \mu] \leq \alpha$, and thus $\mu / \alpha$ is a nonzero Abelian congruence of $\mathbf{C} / \alpha(\cong \mathbf{A})$.

Lemma 4.7. If $\mathscr{L}$ and $\mathscr{K}$ are relatively modular quasivarieties and $\mathscr{L} \subseteq \mathscr{K}$, then $\mathscr{L}_{\mathrm{FSI}}$ is closed under similarity in $\mathscr{K}$.

Proof. Assume that $\mathbf{A} \in \mathscr{L}_{\mathrm{FSI}}, \mathbf{B} \in \mathscr{K}_{\mathrm{FSI}} \cap \boldsymbol{H}(\mathscr{L})$, and $\mathbf{C}, \alpha, \alpha^{*}, \beta, \beta^{*}, \delta$, $\gamma$ witness that $\mathbf{A} \sim \mathscr{K}$ B . As noted earlier, we can choose $\mathbf{C}$ so that $\alpha \beta=0_{C}$, hence $\mathbf{C}$ belongs to the variety $\boldsymbol{H}(\mathscr{L})$. Then making another choice of $\mathbf{C}$, we can assume that $\mathbf{C} \in \mathscr{L}$. Now $\alpha \in \operatorname{Con}_{\mathscr{L}} \mathbf{C}$; i.e., $\alpha=\mathrm{Cg}_{\mathscr{L}}(\alpha)$. We need to show that $\beta=\operatorname{Cg}_{\mathscr{L}}(\beta)$. To get a contradiction, suppose that $\beta<$ $\mathrm{Cg}_{\mathscr{L}}(\beta)$. Then of course $\mathrm{Cg}_{\mathscr{L}}(\beta) \beta^{*}>\beta$. Replacing $\beta^{*}$ by $\mathrm{Cg}_{\mathscr{L}}(\beta) \beta^{*}, \gamma$ by $\operatorname{Cg}_{\mathscr{L}}(\beta) \gamma \beta^{*}$, and $\alpha^{*}$ by $\alpha+\mathscr{K} \mathrm{Cg}_{\mathscr{L}}(\beta) \gamma \beta^{*}$, we can change notation and assume that $\operatorname{Cg}_{\mathscr{L}}(\beta) \geq \beta^{\star} \geq \gamma$. By the extension principle, we now have

$$
\mathrm{Cg}_{\mathscr{L}}(\boldsymbol{\delta})=\mathrm{Cg}_{\mathscr{L}}(\gamma \beta)=\mathrm{Cg}_{\mathscr{L}}(\gamma) \mathrm{Cg}_{\mathscr{L}}(\beta)=\mathrm{Cg}_{\mathscr{L}}(\gamma) ;
$$

then

$$
\begin{aligned}
\operatorname{Cg}_{\mathscr{L}}\left(\alpha^{\star}\right) & =\operatorname{Cg}_{\mathscr{L}}(\alpha+\mathscr{K} \gamma)=\mathrm{Cg}_{\mathscr{L}}(\alpha)+{ }_{\mathscr{L}} \mathrm{Cg}_{\mathscr{L}}(\gamma) \\
& =\operatorname{Cg}_{\mathscr{L}}(\alpha)+\mathscr{L} \operatorname{Cg}_{\mathscr{L}}(\delta)=\operatorname{Cg}_{\mathscr{L}}(\alpha+\delta)=\operatorname{Cg}_{\mathscr{L}}(\alpha)=\alpha .
\end{aligned}
$$

This is a contradiction, since $\alpha<\alpha^{\star} \leq \mathrm{Cg}_{\mathscr{L}}\left(\alpha^{\star}\right)$.

Theorem 4.8. Assume that $\mathscr{K}$ is a relatively modular quasivariety and that $\mathscr{L} \subseteq$ $\mathscr{K}$ is a subquasivariety. Then the following conditions are equivalent:

(1) $\mathscr{L}$ is relatively modular.

(2) $\mathscr{L}$ satisfies the extension principle.

(3) (i) $\mathscr{L}_{\mathrm{RSI}} \subseteq \mathscr{L}_{\mathrm{FSI}}$ and(ii) $\mathscr{L}_{\mathrm{FSI}}$ is closed under similarity in $\mathscr{K}$.

Proof. Theorem 4.1 easily implies that conditions (1) and (2) are equivalent. Further, from Lemma 4.7 and earlier work, we already know that conditions (3)(i) and (3)(ii) are necessary conditions for $\mathscr{L}$ to be relatively modular. Suppose that these conditions hold. We shall prove that (2) holds.

Arguing by contradiction, we suppose instead that (2) fails. We have $\mathbf{A} \in \mathscr{L}$ and congruences $\alpha$ and $\beta$ of $\mathbf{A}$ such that $\mathrm{Cg}_{\mathscr{L}}(\alpha \beta)<\mathrm{Cg}_{\mathscr{L}}(\alpha) \mathrm{Cg}_{\mathscr{L}}(\beta)$. Since $\mathscr{K}$ satisfies the extension principle,

$$
\mathrm{Cg}_{\mathscr{L}}\left(\mathrm{Cg}_{\mathscr{H}}(\alpha) \mathrm{Cg}_{\mathscr{H}}(\beta)\right)=\mathrm{Cg}_{\mathscr{L}}(\alpha \beta)<\mathrm{Cg}_{\mathscr{L}}\left(\mathrm{Cg}_{\mathscr{H}}(\alpha)\right) \mathrm{Cg}_{\mathscr{L}}\left(\mathrm{Cg}_{\mathscr{H}}(\beta)\right) \text {. }
$$

Replacing $\alpha$ and $\beta$ by their $\mathscr{K}$-extensions if necessary, we may assume that they are $\mathscr{K}$-congruences.

There is a congruence $\mu \in \operatorname{Con}_{\mathscr{L}} \mathbf{A}$ which is completely meet-irreducible in $\operatorname{Con}_{\mathscr{L}} \mathbf{A}$ such that $\mu \geq \alpha \beta$ and $\mu \geq \mathrm{Cg}_{\mathscr{L}}(\alpha) \mathrm{Cg}_{\mathscr{L}}(\beta)$. We can assume that $\alpha$ and $\beta$ are maximal with respect to being $\mathscr{K}$-congruences and satisfying 
$\alpha \beta \leq \mu$, i.e., that if $\alpha^{\star}$ is a $\mathscr{K}$-congruence and $\alpha^{\star}>\alpha$ then $\alpha^{\star} \beta \leq \mu$, and similarly, if $\beta^{\star}$ is a $\mathscr{K}$-congruence and $\beta^{\star}>\beta$ then $\alpha \beta^{\star} \leq \mu$. Clearly, neither $\alpha$ nor $\beta$ is contained in $\mu$. (If $\alpha \leq \mu$ then $\operatorname{Cg}_{\mathscr{L}}(\alpha) \leq \operatorname{Cg}_{\mathscr{L}}(\mu)=\mu$ which is false.)

Since $\alpha(\mu \alpha+\mathscr{K} \beta)=\mu \alpha+\mathscr{K} \alpha \beta \leq \mu$ holds by modularity, it follows from the maximality of $\beta$ that $\mu \alpha \leq \beta$. Similar arguments give that

$$
\alpha \beta=\alpha \mu=\beta \mu \text {. }
$$

Now $\mu$ is meet-irreducible, by $(3)(\mathrm{i})$. Hence

$$
\lambda=(\mu+\mathscr{K} \alpha)(\mu+\mathscr{R} \beta)>\mu \text {. }
$$

We have $\lambda / \mu \searrow \gamma / \alpha \beta$ in $\operatorname{Con}_{\mathscr{H}} \mathbf{A}$, where $\gamma=(\mu+\mathscr{H} \alpha) \beta$. Moreover, we have $\alpha^{\star} / \alpha \searrow \gamma / \alpha \beta$, where $\alpha^{\star}=\alpha+\mathscr{K} \gamma$. Thus $\alpha^{\star} / \alpha \searrow \gamma / \alpha \beta \nearrow \lambda / \mu$ in $\operatorname{Con}_{\mathscr{K}} \mathbf{A}$.

We can now show that $\alpha$ is meet-irreducible as an ordinary congruence. For this, it will suffice to show that $\alpha$ is meet-irreducible in $\operatorname{Con}_{\mathscr{K}} \mathbf{A}$, since $\mathscr{K}$ satisfies the extension principle. From (3)(i), $\mu$ is meet-irreducible as an ordinary congruence and therefore as a $\mathscr{K}$-congruence. This fact and the $\mathscr{K}$ isotopy shown in the last displayed formula show that $\alpha$ is meet-irreducible in the interval $\alpha^{\star} / \alpha$ of $\operatorname{Con}_{\mathscr{K}} \mathbf{A}$. Thus we need only show that if $\chi \in \operatorname{Con}_{\mathscr{K}} \mathbf{A}$ and $\chi>\alpha$ then $\chi \alpha^{\star}>\alpha$. Assume that $\chi>\alpha$. By the maximality of $\alpha$, we have that $\chi \beta \not \mu \mu$. Hence $\chi \beta+_{\mathscr{K}} \mu>\mu$, and it follows that $\lambda(\chi \beta+\mathscr{K} \mu)>\mu$. Working back through the isotopies, we have that

$$
\alpha+\mathscr{K} \gamma \lambda(\chi \beta+\mathscr{K} \mu)>\alpha .
$$

Since $\lambda \geq \mu, \lambda \beta=\gamma$, and $\gamma \mu=\alpha \beta$, the displayed inequality can be rewritten (using the modular law) as $\gamma \chi \not \leq \alpha$. This implies $\alpha^{\star} \chi \leq \underline{\alpha}$, since $\alpha^{\star} \geq \gamma$. Thus $\alpha$ is meet-irreducible as a $\mathscr{K}$-congruence and also as an ordinary congruence.

Now the displayed isotopies show that the finitely subdirectly irreducible algebras $\mathbf{A} / \alpha$ and $\mathbf{A} / \mu$ are $\mathscr{K}$-similar; hence we conclude by (3)(ii) that $\mathbf{A} / \alpha \in$ $\mathscr{L}$, and so $\alpha=\mathrm{Cg}_{\mathscr{L}}(\alpha)$. A similar argument gives that $\beta=\mathrm{Cg}_{\mathscr{L}}(\beta)$. This of courses contradicts our assumptions that $\alpha \beta \leq \mu$ while $\operatorname{Cg}_{\mathscr{L}}(\alpha) \operatorname{Cg}_{\mathscr{L}}(\beta) \not$ $\mu$.

One can use Theorem 4.8(3)(ii) and the remarks preceding Lemma 4.7 to show that, in Theorem $4.8, \mathscr{L}$ is a relatively distributive subquasivariety of $\mathscr{K}$ iff $\mathscr{L}_{\text {RSI }} \subseteq \mathscr{L}_{\text {FSI }}$ and $\mathscr{L} \models_{\text {con }}[x, x]_{\mathscr{K}}=0 \rightarrow x=0$. This was proved in [K2] under the assumption that $\mathscr{K}$ is a variety. If, in Theorem $4.8, \mathscr{K}$ is relatively distributive, then $\mathscr{L}$ is relatively modular iff it is relatively distributive iff $\mathscr{L}_{\text {RSI }} \subseteq \mathscr{L}_{\text {FSI }}$. This extends the result that $\mathscr{L}$ is a relatively distributive subquasivariety of a congruence distributive variety iff $\mathscr{L}_{\text {RSI }} \subseteq \mathscr{L}_{\text {FSI }}$, which was proved in [Dz2].

\section{AN ALMOST-EQUATIONAL AXIOMATIZATION}

By a $\Delta$-axiom we mean a first order sentence of the form "for all $x, \ldots, \bar{z}$ we have $p_{i}(x, x, \bar{u}, \bar{u}, \bar{z})=q_{i}(x, x, \bar{u}, \bar{u}, \bar{z})$ for all $i \in\{0, \ldots, n-1\}$, and if $p_{i}(x, y, \bar{u}, \bar{u}, \bar{z})=q_{i}(x, y, \bar{u}, \bar{u}, \bar{z})$ for all $i \in\{0, \ldots, n-1\}$ then $x=y$," for some finite system of terms $p_{i}(x, y, \bar{u}, \bar{v}, \bar{z}), q_{i}(x, y, \bar{u}, \bar{v}, \bar{z})$. We write this axiom as $\Delta\left(p_{i}, q_{i} ; 0 \leq i<n\right)$, or simply $\Delta(p, q)$. If we have a quasi-equation

$$
\bigwedge_{0 \leq j<m} r_{j}(\bar{z}) \approx s_{j}(\bar{z}) \rightarrow r(\bar{z}) \approx s(\bar{z})
$$


and a $\Delta$-axiom $\Delta(p, q)$, where $p=p(x, y, \bar{u}, \bar{v}, \bar{z})$ and $\bar{u}, \bar{v}$ are $m$-tuples of variables, then obviously this quasi-equation is implied by $\Delta(p, q)$ together with the system of equations

$$
\begin{aligned}
& p_{i}\left(r(\bar{z}), s(\bar{z}), r_{0}(\bar{z}), \ldots, r_{m-1}(\bar{z}), s_{0}(\bar{z}), \ldots, s_{m-1}(\bar{z}), \bar{z}\right) \\
& \quad \approx q_{i}\left(r(\bar{z}), s(\bar{z}), r_{0}(\bar{z}), \ldots, r_{m-1}(\bar{z}), s_{0}(\bar{z}), \ldots, s_{m-1}(\bar{z}), \bar{z}\right) .
\end{aligned}
$$

This observation leads to the next theorem.

Theorem 5.1. Let $\mathscr{K}$ be a relatively modular quasivariety. $\mathscr{K}$ is axiomatized by a set of $\Delta$-axioms combined with a set of equations.

Proof. Suppose that $\bigwedge_{j<m} r_{j} \approx s_{j} \rightarrow r \approx s$ is one of the $k$-variable axioms of $\mathscr{K}$. Let $\mathbf{F}$ be the $\mathscr{K}$-free algebra generated by $z_{j}(j<k)$ together with $x, y, u_{0}, \ldots, u_{m-1}, v_{0}, \ldots, v_{m-1}$. Consider the congruences $\gamma$ generated by all pairs $(x, y),\left(u_{j}, v_{j}\right) ; \alpha$ generated by all pairs $\left(u_{j}, v_{j}\right)$; and $\beta$ generated by all pairs $(x, r(\bar{z})),(y, s(\bar{z})),\left(u_{j}, r_{j}(\bar{z})\right)$, and $\left(v_{j}, s_{j}(\bar{z})\right)$. Then $(x, y)$ belongs to

$$
\gamma^{\prime}\left(\alpha^{\prime}+\beta^{\prime}\right)=(\alpha+\gamma \beta)^{\prime}
$$

by the modularity and the extension principle. So we get finitely many pairs $\left(p_{i}, q_{i}\right)$ of elements of $\gamma \beta$ such that $(x, y)$ is in every $\mathscr{K}$-congruence containing these pairs and $\alpha$. This just means that

$$
\begin{aligned}
\mathscr{K} \models & p_{i}\left(r(\bar{z}), s(\bar{z}), r_{0}(\bar{z}), \ldots, s_{m-1}(\bar{z}), \bar{z}\right) \\
& \approx q_{i}\left(r(\bar{z}), s(\bar{z}), r_{0}(\bar{z}), \ldots, s_{m-1}(\bar{z}), \bar{z}\right)
\end{aligned}
$$

and

$$
\mathscr{K} \models p_{i}(x, x, \bar{u}, \bar{u}, \bar{z}) \approx q_{i}(x, x, \bar{u}, \bar{u}, \bar{z})
$$

for all $i ;$ and

$$
\mathscr{K} \models\left(\bigwedge_{i} p_{i}(x, y, \bar{u}, \bar{u}, \bar{z}) \approx q_{i}(x, y, \bar{u}, \bar{u}, \bar{z})\right) \rightarrow x \approx y .
$$

Thus the $\Delta$-axiom $\Delta(p, q)$ holds in $\mathscr{K}$; and, combined with the first set of equations displayed above, it implies the given quasi-equation. This establishes the theorem.

(The referee informs us that Theorem 5.1 for relatively distributive quasivarieties is Theorem 4.1 in The deduction-like theorem for quasivarieties of algebras and its applications, by J. Czelakowski and W. Dziobiak which will appear in Algebraic Logic (Proc. Conf. Budapest, 1988): (H. Andréka, J.D. Monk, and I. Németi, eds.), Colloq. Math. Soc. János Bolyai, North-Holland, Amsterdam.)

The quasivariety of all cancellation semigroups, $\mathscr{C}$, is axiomatized by the quasi-equations

$$
x y \approx x z \rightarrow y \approx z \text { and } y x \approx z x \rightarrow y \approx z .
$$

Each of these quasi-equations can easily be written as a $\Delta$-axiom. However, the quasivariety of all cancellation semigroups is not relatively modular; it does not even satisfy the extension principle. For example, in $\mathbf{F}_{\leftarrow}(x, y, z, u)$ we have $\mathrm{Cg}(x z, x u) \cap \mathrm{Cg}(y z, y u)=0$, but $(z, u) \in \mathrm{Cg}_{\nprec}(x z, x u) \cap \mathrm{Cg}_{\leftarrow}(y z, y u)$. 
Theorem 5.2. Let $\mathscr{K}$ be a relatively modular quasivariety. Let $\mathbf{A} \in \mathscr{K}, \theta \in$ Con $\mathbf{A}$, and $u, v \in A$. Then $(u, v)$ belongs to the $\mathscr{K}$-extension of $\theta$ iff there is some $\Delta$-axiom $\Delta(p, q)$ valid in $\mathscr{K}$, some pairs $\left(a_{i}, b_{i}\right) \in \theta$, and some elements $\bar{c} \in \mathbf{A}$ such that $p_{j}^{\mathbf{A}}(u, v, \bar{a}, \bar{b}, \bar{c})=q_{j}^{\mathbf{A}}(u, v, \bar{a}, \bar{b}, \bar{c})$ for all $i$.

Proof. It is obvious that the condition implies that $(u, v)$ belongs to the $\mathscr{K}$ extension of $\theta$. Conversely, suppose that $(u, v) \in \mathrm{Cg}_{\mathscr{H}}(\theta)$. Let $X=A$ and $\mathbf{F}=\mathbf{F}_{\mathscr{K}}(X)$. Let $\hat{\theta}=\varphi^{-1}(\theta)$, where $\varphi$ is the obvious homomorphism from $\mathbf{F}$ onto $\mathbf{A}$ (that extends the identity map on $X$ ). Then $\operatorname{Cg}_{\mathscr{H}}(\hat{\theta})=$ $\varphi^{-1}\left(\mathrm{Cg}_{\mathscr{K}}(\theta)\right)$, and so $(u, v) \in \mathrm{Cg}_{\mathscr{K}}(\hat{\theta})$. This means that there exist finitely many pairs $\left(r_{i}, s_{i}\right) \in \hat{\theta}, 0 \leq i<m$, such that

$$
\mathscr{K} \models\left(\bigwedge_{i} r_{i} \approx s_{i}\right) \rightarrow r \approx s,
$$

where, for a certain finite system $\bar{z}$ of free generators of $\mathbf{F}$ excluding $u$ and $v$, we have

$$
r_{i}=r_{i}(u, v, \bar{z}), \quad s_{i}=s_{i}(u, v, \bar{z}), \quad r=u, \quad s=v .
$$

Now the proof of Theorem 5.1 produces a $\Delta$-axiom $\Delta(p, q)$ satisfied by $\mathscr{K}$ such that the equations

$$
\begin{aligned}
& p_{j}\left(u, v, r_{0}(u, v, \bar{z}), \ldots, s_{m-1}(u, v, \bar{z}), u, v, \bar{z}\right) \\
& \quad \approx q_{j}\left(u, v, r_{0}(u, v, \bar{z}), \ldots, s_{m-1}(u, v, \bar{z}), u, v, \bar{z}\right)
\end{aligned}
$$

are valid in $\mathscr{K}$. Letting $\bar{a}$ be the tuple $\left(r_{0}^{\mathbf{A}}(u, v, \bar{z}), \ldots, r_{m-1}^{\mathbf{A}}(u, v, \bar{z})\right)$ and $\bar{b}$ the corresponding tuple formed by the evaluations of the $s_{i}$ in $\mathbf{A}$, we have that $a_{i} \theta b_{i}$ for $0 \leq i<m$ since $\left(r_{i}, s_{i}\right) \in \hat{\theta}$; and we have

$$
p_{j}^{\mathbf{A}}(u, v, \bar{a}, \bar{b}, u, v, \bar{z})=q_{j}^{\mathbf{A}}(u, v, \bar{a}, \bar{b}, u, v, \bar{z})
$$

for all $j$ as desired.

\section{THE LATTICE OF RELATIVELY MODULAR QUASIVARIETIES}

The lattice of subvarieties of a modular variety is a modular lattice. The lattice of subvarieties of a distributive variety is a distributive lattice. Indeed, the lattice of subvarieties of any variety is dually isomorphic to a sublattice of the congruence lattice of a free algebra in the variety; so if a variety satisfies a lattice equation as a congruence identity, then the lattice of subvarieties satisfies the dual equation. In this section, we prove that the relatively modular subquasivarieties of a given relatively modular quasivariety constitute a lattice; and we study that lattice. We shall find that it is not possible to identify this lattice with a sublattice of the dual of a relative congruence lattice of any member of the quasivariety.

Lemma 6.1. If $\mathscr{K}_{i}(i \in I)$ are quasivarieties satisfying the extension principle, then $\bigcap_{i \in I} \mathscr{K}_{i}$ satisfies the extension principle. If $\mathscr{K}$ and $\mathscr{L}$ satisfy the extension principle and $\boldsymbol{H}(\mathscr{K})=\boldsymbol{H}(\mathscr{L})$, then the quasivariety join $\mathscr{K} \vee \mathscr{L}$ satisfies the extension principle.

Proof. Let $\mathscr{K}=\bigcap_{i \in I} \mathscr{K}_{i}$ and choose $\mathbf{A} \in \mathscr{K}$. Note that for any quasivariety $\mathscr{L}$, since $\mathrm{Cg}_{\mathscr{L}}$ is an algebraic closure operator on $A^{2}, \operatorname{Con}_{\mathscr{L}} \mathbf{A}$ is closed under 
unions of up-directed sets of congruences. Now suppose that $\mathbf{A} \in \mathscr{L} \cap \mathscr{J}$ and both $\mathrm{Cg}_{\mathscr{L}}$ and $\mathrm{Cg}_{\mathscr{J}}$ are meet-preserving maps on Con $\mathrm{A}$. We shall show that $\mathrm{Cg}_{\mathscr{L} \cap \mathcal{J}}$ is meet-preserving likewise. For $\theta \in \operatorname{Con} \mathbf{A}$ and $n<\omega$ define

$$
\theta_{n}= \begin{cases}\theta & \text { if } n=0, \\ \operatorname{Cg}_{\mathscr{L}}\left(\theta_{n-1}\right) & \text { if } n \text { is odd, } \\ \operatorname{Cg}_{\mathscr{J}}\left(\theta_{n-1}\right) & \text { otherwise. }\end{cases}
$$

Then $\bigvee_{n<\omega} \theta_{n}$ belongs to both $\operatorname{Con}_{\mathscr{L}} \mathbf{A}$ and $\operatorname{Con}_{\mathscr{f}} \mathbf{A}$ (since both sets are closed under up-directed unions); so clearly,

$$
\bigvee_{n<\omega} \theta_{n}=\operatorname{Cg}_{\mathscr{L} \cap \mathscr{J}}(\theta) .
$$

If $\gamma, \delta \in$ Con $\mathbf{A}$, then for every $n<\omega$ we have $\gamma_{n} \cdot \delta_{n}=(\gamma \cdot \delta)_{n}$, since both $\mathrm{Cg}_{\mathscr{L}}$ and $\mathrm{Cg}_{\mathscr{f}}$ preserve meets. By upper continuity, this entails that

$$
\left(\bigvee_{n<\omega} \gamma_{n}\right) \cdot\left(\bigvee_{n<\omega} \delta_{n}\right)=\bigvee_{n<\omega}(\gamma \cdot \delta)_{n},
$$

or that

$$
\mathrm{Cg}_{\mathscr{L} \cap \mathcal{J}}(\gamma \cdot \delta)=\mathrm{Cg}_{\mathscr{L} \cap \mathcal{J}}(\gamma) \cdot \mathrm{Cg}_{\mathscr{L} \cap \mathcal{F}}(\delta) .
$$

$\gamma$ and $\delta$ were arbitrary, so we have shown that $\mathrm{Cg}_{\mathscr{L} \cap \mathscr{J}}$ preserves meets.

Take $\Gamma$ to be the set of all maps $\mathrm{Cg}_{\mathscr{L}}$, where $\mathscr{L}$ ranges over intersections of finite subfamilies of $\left\{\mathscr{K}_{i}: i \in I\right\}$ and $\mathrm{Cg}_{\mathscr{L}}$ is construed as a function from Con $\mathbf{A}$ into Con $\mathbf{A}$. From the previous paragraph, we have that each member of $\Gamma$ is a meet-preserving function. We claim that for $\theta \in \operatorname{Con} \mathbf{A}$,

$$
\mathrm{Cg}_{\mathscr{K}}(\theta)=\bigvee_{C \in \Gamma} C(\theta)=\bigcup_{C \in \Gamma} C(\theta) \text {. }
$$

The second equality holds because $\{C(\theta): C \in \Gamma\}$ is an up-directed set of congruences. Also, it is clear that $\operatorname{Cg}_{\mathscr{K}}(\theta) \geq C(\theta)$ when $C \in \Gamma$, since $C=$ $\mathrm{Cg}_{\mathscr{L}}$, where $\mathscr{L} \supseteq \mathscr{K}$. The first equality in the displayed formula can thus be demonstrated by showing that $\bigvee\{C(\theta): C \in \Gamma\}$ belongs to $\operatorname{Con}_{\mathscr{H}} \mathbf{A}$. To do this, let $i \in I$, and note that since $\operatorname{Cg}_{\mathscr{L}}(\theta) \leq \operatorname{Cg}_{\mathscr{L} \cap \mathscr{H}_{i}}(\theta)$, then

$$
\bigvee\{C(\theta): C \in \Gamma\}=\bigvee\left\{C(\theta): C \in \Gamma \text { and } C(\theta) \in \operatorname{Con}_{\mathscr{H}_{i}} \mathbf{A}\right\}
$$

This displays the join as equal to the join of an up-directed subset of $\mathrm{Con}_{\mathscr{F}_{i}} \mathbf{A}$; consequently, the join belongs to $\operatorname{Con}_{\mathscr{H}_{i}} \mathbf{A}$. Since this holds for every $i$, we conclude that $\bigvee\{C(\theta): \theta \in C\}$ belongs to $\operatorname{Con}_{\mathscr{H}} \mathbf{A}$, as desired.

Now if $(x, y) \in \mathrm{Cg}_{\mathscr{R}}(\theta) \mathrm{Cg}_{\mathscr{H}}(\psi)$, where $\theta, \psi \in \operatorname{Con} \mathbf{A}$, then, as we have shown above, $(x, y) \in \mathrm{Cg}_{\mathscr{L}}(\theta) \cap \mathrm{Cg}_{\mathscr{J}}(\psi)$ for some $\mathscr{L}$ and $\mathscr{J}$ which are intersections of finitely many of the $\mathscr{K}_{i}$. So we have

$$
(x, y) \in \mathrm{Cg}_{\mathscr{L}^{\prime}}(\theta) \mathrm{Cg}_{\mathscr{L}^{\prime}}(\psi)=\mathrm{Cg}_{\mathscr{L}^{\prime}}(\theta \psi) \subseteq \mathrm{Cg}_{\mathscr{K}}(\theta \psi),
$$

where $\mathscr{L}^{\prime}=\mathscr{L} \cap \mathscr{J}$, since $\mathrm{Cg}_{\mathscr{L}}$ is meet-preserving, as was shown in the first paragraph of the proof. This concludes the proof of the first statement in Lemma 6.1. 
To prove the second statement, first notice that $\mathscr{K} \vee \mathscr{L}=\boldsymbol{S P P}_{u}(\mathscr{K} \cup \mathscr{L})$ $=\boldsymbol{P}_{s}(\mathscr{K} \cup \mathscr{L})$. This implies that for $\mathbf{A} \in \mathscr{K} \cap \mathscr{L}$ and $\theta \in$ Con $\mathbf{A}$ we have the formula

$$
\mathrm{Cg}_{\mathscr{K} \vee \mathscr{L}}(\theta)=\mathrm{Cg}_{\mathscr{K}}(\theta) \cdot \mathrm{Cg}_{\mathscr{L}}(\theta)
$$

Now, $\mathscr{K}$ and $\mathscr{L}$ satisfy the extension principle iff their free members do. Further, $\boldsymbol{H}(\mathscr{K})=\boldsymbol{H}(\mathscr{L})$ holds iff $\mathscr{K}$, and $\mathscr{L}$ have the same free algebras. Thus $\mathscr{K}, \mathscr{L}$ and $\mathscr{K} \vee \mathscr{L}$ have the same free algebras. Hence it suffices to consider an arbitrary free algebra $\mathbf{F} \in \mathscr{K} \cap \mathscr{L}$ and show that for congruences on $\mathbf{F}$ we have $\operatorname{Cg}_{\mathscr{K} \vee \mathscr{L}}(\alpha \cdot \beta)=\mathrm{Cg}_{\mathscr{H} \vee \mathscr{L}}(\alpha) \cdot \mathrm{Cg}_{\mathscr{H} \vee \mathscr{L}}(\beta)$. By the last displayed formula,

$$
\begin{aligned}
\mathrm{Cg}_{\mathscr{K} \vee \mathscr{L}}(\alpha \cdot \beta) & =\mathrm{Cg}_{\mathscr{H}}(\alpha \cdot \beta) \cdot \mathrm{Cg}_{\mathscr{L}}(\alpha \cdot \beta) \\
& =\mathrm{Cg}_{\mathscr{H}}(\alpha) \cdot \mathrm{Cg}_{\mathscr{H}}(\beta) \cdot \mathrm{Cg}_{\mathscr{L}}(\alpha) \cdot \mathrm{Cg}_{\mathscr{L}}(\beta) \\
& =\mathrm{Cg}_{\mathscr{H} \vee \mathscr{L}}(\alpha) \cdot \mathrm{Cg}_{\mathscr{H} \vee \mathscr{L}}(\beta) .
\end{aligned}
$$

This proves the second statement of the lemma.

Lemma 6.1 and Theorem 4.8 show that the family of all relatively modular subquasivarieties of a given relatively modular quasivariety is closed under arbitrary intersections. This implies that the inclusion ordering for this family is a lattice ordering. If $\mathscr{K}$ is relatively modular, let $\mathbf{L}(\mathscr{K})$ and $\mathbf{L}_{\mathbf{M}}(\mathscr{K})$ denote the lattices of subquasivarieties of $\mathscr{K}$, and of relatively modular subquasivarieties of $\mathscr{K}$, respectively.

We observed above that two quasivarieties which generate the same variety must possess the same free algebras. Consequently, the family of quasivarieties generating a given variety is closed under arbitrary intersections.

Corollary 6.2. If $\mathscr{V}$ is a variety, then the lattice of all quasivarieties which generate $\mathscr{V}$ and satisfy the extension principle is a distributive sublattice of the lattice of subquasivarieties of $\mathscr{V}$.

Proof. Lemma 6.1 insures that the quasivarieties which generate $\mathscr{V}$ and satisfy the extension principle do indeed form a sublattice of the lattice of subquasivarieties of $\mathscr{V}$. Suppose that $\mathscr{J}, \mathscr{K}$, and $\mathscr{L}$ are three members of this sublattice and that $\mathscr{M}=\mathscr{J} \cap(\mathscr{K} \vee \mathscr{L})$. Then

$$
\begin{aligned}
\mathscr{M}_{\mathrm{FSI}} & =\mathscr{F}_{\mathrm{FSI}} \cap\left(\mathscr{K}_{\mathrm{FSI}} \cup \mathscr{L}_{\mathrm{FSI}}\right) \\
& =\left(\mathscr{F}_{\mathrm{FSI}} \cap \mathscr{K}_{\mathrm{FSI}}\right) \cup\left(\mathscr{L}_{\mathrm{FSI}} \cap \mathscr{L}_{\mathrm{FSI}}\right) \subseteq(\mathscr{J} \cap \mathscr{K}) \cup(\mathscr{J} \cap \mathscr{L}) .
\end{aligned}
$$

But $\mathscr{M}$ satisfies the extension principle, so $\mathscr{M}_{\mathrm{RSI}} \subseteq \mathscr{M}_{\mathrm{FSI}}$ which implies that $\mathscr{M}=\boldsymbol{P}_{s}\left(\mathscr{M}_{\mathrm{FSI}}\right)$. Hence, $\mathscr{M} \subseteq(\mathscr{J} \cap \mathscr{K}) \vee(\mathscr{J} \cap \mathscr{L})$.

Every quasivariety $\mathscr{J}$ is contained in a quasivariety that satisfies the extension principle: the variety generated by $\mathcal{J}$, for example. Lemma 6.1 implies that there is a least quasivariety $\bar{J}$ containing $\mathscr{J}$ that satisfies the extension principle. Observe that if $\mathcal{J}$ is contained in any relatively modular quasivariety then, by Theorem $4.8, \bar{J}$ is the least relatively modular quasivariety containing $\mathscr{J}$. It may be that $\bar{J}$ may be described as the least quasivariety containing $\mathcal{J}$ which can be axiomatized by special sorts of axioms; axioms more special than quasi-equations but less special than equations. Or it may be that $\bar{J}$ can be described as the closure of $\mathscr{J}$ under some class operator; closing $\mathcal{J}$ under a limited kind of homomorphism. We do not know if either of these guesses is true or not, but we will digress a little from the examination of the 
lattice $\mathbf{L}_{\mathbf{M}}(\mathscr{K})$ and show that quasivarieties satisfying the extension principle are closed under a kind of "factorization" of algebras.

Definition 6.1. If $f: \mathbf{B} \rightarrow \mathbf{D}$ and $g: \mathbf{C} \rightarrow \mathbf{D}$ are onto homomorphisms, then we will write $\mathbf{B} \times{ }_{\mathbf{D}} \mathbf{C}$ to denote the subalgebra of $\mathbf{B} \times \mathbf{C}$ consisting of all pairs $(b, c)$ for which $f(b)=g(c)$.

Theorem 6.3 (Factorization Theorem). If $\mathscr{K}$ satisfies the extension principle, $\mathbf{B} \times_{\mathbf{D}} \mathbf{C} \in \mathscr{K}$, and $\mathbf{D} \in \mathscr{K}$, then both $\mathbf{B}$ and $\mathbf{C}$ are members of $\mathscr{K}$. In particular, if $\mathbf{B} \times \mathbf{C}$ belongs to $\mathscr{K}$, then both $\mathbf{B}$ and $\mathbf{C}$ also do.

Proof. Let $\eta_{0}$ and $\eta_{1}$ denote the congruences on $\mathbf{B} \times_{\mathbf{D}} \mathbf{C}$ that are the kernels of the projections onto $\mathbf{B}$ and $\mathbf{C}$ respectively. Now if $\alpha$ is the kernel of $f$ and $\beta$ is the kernel of $g$, then $\alpha_{0}=\beta_{1}=\eta_{0}+\eta_{1}$, which is a $\mathscr{K}$-congruence since $\mathbf{D} \in \mathscr{K}$. Further, $\eta_{0} \eta_{1}=0$ is a $\mathscr{K}$-congruence since $\mathbf{B} \times_{\mathbf{D}} \mathbf{C}$ is in $\mathscr{K}$. Our goal is to prove that both $\eta_{0}$ and $\eta_{1}$ are $\mathscr{K}$-congruences. We have $\eta_{0} \leq \eta_{0}^{\prime} \leq \eta_{0}+\eta_{1}$. Assume that $\langle(b, c),(d, e)\rangle \in \eta_{0}^{\prime}-\eta_{0}$. Then $b \neq d$, but $f(b)=f(d)=g(c)=g(e)$. This implies that $\langle(b, c),(d, c)\rangle \in \eta_{0}^{\prime} \eta_{1}^{\prime}$, which contradicts the fact that $\eta_{0}^{\prime} \eta_{1}^{\prime}=0$. We must conclude that $\eta_{0}=\eta_{0}^{\prime}$ and similarly that $\eta_{1}=\eta_{1}^{\prime}$.

The second statement follows from the first statement and the fact that $\mathbf{B} \times \mathbf{C}$ is just $\mathbf{B} \times_{\mathbf{D}} \mathbf{C}$ for $\mathbf{D}$ equal to the one-element algebra, which is a member of $\mathscr{K}$.

The quasivariety of all cancellation semigroups is closed under the kind of factorization described here, but this quasivariety does not satisfy the extension principle, as we pointed out after Theorem 5.1.

The next result is the quasivariety analogue of a well-known result of J. Hagemann and C. Herrmann (cf. Exercise 8.2 in [FM]).

Theorem 6.4. The quasivariety join of two relatively distributive subquasivarieties of a relatively modular quasivariety is relatively distributive.

Proof. Let $\mathscr{K}$ be relatively modular and assume that $\mathscr{J}$ and $\mathscr{L}$ are relatively distributive subquasivarieties of $\mathscr{K}$. Let $\mathscr{M}$ be the quasivariety join $\mathscr{J} \vee \mathscr{L}$. We will prove that $\mathscr{M}$ is relatively distributive by using the criteria mentioned after Theorem 4.8; that is, by showing that $\mathscr{M}_{\mathrm{RSI}} \subseteq \mathscr{M}_{\mathrm{FSI}}$ and that $\mathscr{M}$ satisfies the commutator implication $[x, x]_{\mathscr{K}}=0 \rightarrow x=0$.

From $\mathscr{J} \vee \mathscr{L}=\boldsymbol{P}_{S}(\mathscr{J} \cup \mathscr{L})$ we find that $\mathscr{M}_{\mathrm{RSI}} \subseteq \mathscr{J}_{\mathrm{RSI}} \cup \mathscr{L}_{\mathrm{RSI}} \subseteq \mathscr{J}_{\mathrm{FSI}} \cup \mathscr{L}_{\mathrm{FSI}}$ $=\mathscr{M}_{\text {FSI }}$. To see that $\mathscr{M}$ satisfies $[x, x]_{\mathscr{K}}=0 \rightarrow x=0$, let $\mathbf{A} \in \mathscr{M}$ and $\theta \in$ Con $\mathbf{A}$ with $[\theta, \theta]_{\mathscr{K}}=0_{A}$. Since $\mathscr{J} \vee \mathscr{L}=\boldsymbol{P}_{s}(\mathscr{J} \cup \mathscr{L})$, there are congruences $\alpha \in \operatorname{Con} g \mathbf{A}$ and $\beta \in \operatorname{Con} \mathscr{L} \mathbf{A}$ such that $\alpha \beta=0_{A}$. Now

$$
[\alpha+\theta, \alpha+\theta]_{\mathscr{K}} \leq \alpha+\mathscr{K}[\theta, \theta]_{\mathscr{K}}=\alpha,
$$

implying that $(\alpha+\theta) / \alpha=\alpha$ since $\mathbf{A} / \alpha \in \mathscr{J}$ and $\mathscr{J}$ satisfies the commutator implication. This means that $\theta \leq \alpha$. Similarly, $\theta \leq \beta$. So $\theta \leq \alpha \beta=0_{A}$. We conclude that $\mathscr{M}$ satisfies the commutator implication, which finishes the proof.

The last result shows that the relatively distributive subquasivarieties of a relatively modular quasivariety $\mathscr{K}$ form a lattice. We will denote this lattice by $\mathbf{L}_{\mathbf{D}}(\mathscr{K})$. 
Theorem 6.5. Let $\mathscr{K}$ be a relatively modular quasivariety. $\quad \mathbf{L}_{\mathbf{D}}(\mathscr{K})$ is a distributive sublattice of both $\mathbf{L}(\mathscr{K})$ and $\mathbf{L}_{\mathbf{M}}(\mathscr{K})$.

Proof. Lemma 6.1 and Theorem 6.4 show that the relatively distributive subquasivarieties of $\mathscr{K}$ form a sublattice of both $\mathbf{L}(\mathscr{K})$ and $\mathbf{L}_{\mathbf{M}}(\mathscr{K})$. We only need to show that $\mathbf{L}_{\mathbf{D}}(\mathscr{K})$ is distributive. For this it will suffice to show that if $\mathscr{J}, \mathscr{L}$, and $\mathscr{M}$ are relatively distributive subquasivarieties of $\mathscr{K}$, then

$$
(\mathscr{M} \cap(\mathscr{J} \vee \mathscr{L}))_{\mathrm{RSI}} \subseteq(\mathscr{M} \cap \mathscr{J}) \cup(\mathscr{M} \cap \mathscr{L}) .
$$

Now just notice that

$$
\begin{aligned}
(\mathscr{M} \cap(\mathscr{J} \vee \mathscr{L}))_{\mathrm{RSI}} & \subseteq(\mathscr{M} \cap(\mathscr{J} \vee \mathscr{L}))_{\mathrm{FSI}} \\
& =\left(\mathscr{M}_{\mathrm{FSI}} \cap \mathscr{J}_{\mathrm{FSI}}\right) \cup\left(\mathscr{M}_{\mathrm{FSI}} \cap \mathscr{L}_{\mathrm{FSI}}\right) .
\end{aligned}
$$

It is not true that $\mathbf{L}_{\mathbf{M}}(\mathscr{K})$ is always modular, nor is it always a sublattice of $\mathbf{L}(\mathscr{K})$, as the following example shows. In this example, we produce a finitely generated modular variety that has nine relatively modular subquasivarieties, all but one of which are subvarieties.

Example 6.6. Our algebras will be groups with two nullary operations, $a$ and $b$, in addition to the identity element, 1 (i.e., they are doubly-pointed groups). A will be the symmetric group on five letters with $a^{\mathbf{A}}$ interpreted as some transposition and $b^{\mathbf{A}}$ as some element of order five. $\mathbf{A}$ is generated by $a^{\mathbf{A}}$ and $b^{\mathbf{A}}$ so $\mathbf{A}$ has no proper subalgebras. Now there are exactly four two-element groups of the type we are interested in, corresponding to the four ways of assigning the values of $a$ and $b$. We will write $\mathbf{B}, \mathbf{B}_{a}, \mathbf{B}_{b}$, and $\mathbf{B}_{a b}$ using subscripts to denote which constants are different from the identity element. Notice that $\mathbf{A}$ has a unique nontrivial congruence $\theta$ and that $\mathbf{A} / \theta \cong \mathbf{B}_{a}$. Let $\mathscr{K}=\boldsymbol{V}\left(\mathbf{A}, \mathbf{B}_{b}\right)$. We claim that the nonmodular lattice in Figure 5 is $\mathbf{L}_{\mathbf{M}}(\mathscr{K})$. Of course, the varieties listed in the figure are relatively modular. Further,

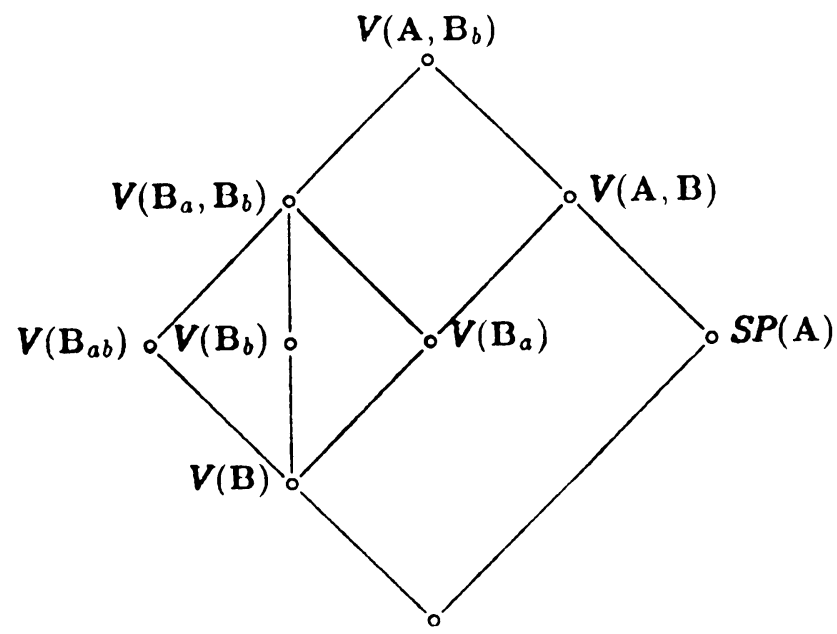

FigURE 5 
$\boldsymbol{S P}(\mathbf{A})$ is relatively distributive by the criteria in Theorem 4.6. What we need to verify is that $\mathbf{L}_{\mathbf{M}}(\mathscr{K})$ contains no other members.

Locally finite, relatively modular quasivarieties are determined by their finite subdirectly irreducible members and $\mathscr{K}$ only contains five subdirectly irreducible algebras: $\mathbf{A}, \mathbf{B}, \mathbf{B}_{a}, \mathbf{B}_{b}$, and $\mathbf{B}_{a b}$. Notice that $\boldsymbol{V}\left(\mathbf{B}_{a}, \mathbf{B}_{b}\right)$ is a locally finite, semisimple modular variety so, by Theorem 1.2, the relatively modular subquasivarieties of this variety are just its subvarieties. Any relatively modular subquasivariety different from one of these must contain $\mathbf{A}$. The quasivariety generated by $\mathbf{A}$ appears in our figure. If a member of $\mathbf{L}_{\mathbf{M}}(\mathscr{K})$ contains $\mathbf{A}$ and a subdirectly irreducible algebra different from $\mathbf{A}$, it must contain $\mathbf{B}$. Since $\mathbf{B}_{a} \in \boldsymbol{H}(\mathbf{A})$ and $\mathbf{B}_{a} \sim \mathscr{K}$, such a quasivariety would also have to contain $\mathbf{B}_{a}$. But the quasivariety generated by $\mathbf{A}, \mathbf{B}$, and $\mathbf{B}_{a}$ is $\boldsymbol{V}(\mathbf{A}, \mathbf{B})$ and we have listed it. Any other member of $\mathbf{L}_{\mathbf{M}}(\mathscr{K})$ must contain all five subdirectly irreducible algebras. From these arguments it follows that $\mathbf{L}_{\mathbf{M}}(\mathscr{K})$ is the lattice in Figure 5 .

This example shows that $\mathbf{L}_{\mathbf{M}}(\mathscr{K})$ need not be modular, and also that it need not be a sublattice of $\mathbf{L}(\mathscr{K})$, since the quasivariety join $\boldsymbol{V}\left(\mathbf{B}_{a}\right) \vee \boldsymbol{V}\left(\mathbf{B}_{b}\right)$ does not contain $\mathbf{B}_{a b}$.

\section{EXAMPLES}

In this section, we present examples of relatively modular quasivarieties, focussing on characterizing the relatively modular quasivarieties of semigroups. The arguments used and the intuition developed make it possible to produce several other examples.

We begin by examining the quasivariety, $\mathscr{K}$, of torsion-free $\mathbf{Z}$-modules, where $\mathbf{Z}$ is the ring of integers. (We call a $\mathbf{Z}$-module torsion-free if it satisfies $n x=0 \rightarrow x=0$ for each nonzero $n \in \mathbf{Z}$.) $\mathscr{K}$ is a relatively modular subquasivariety of the modular variety of all $\mathbf{Z}$-modules, as one may verify by using the criteria of Theorem 4.8. We will discover this fact a different way. If $\mathbf{A} \in \mathscr{H}$ then $\mathbf{Q} \otimes_{\mathbf{Z}} \mathbf{A}$ is a $\mathbf{Q}$-module, where $\mathbf{Q}$ denotes the ring of rational numbers. Further, the $\mathbf{Z}$-linear map,

$$
\eta: \mathbf{A} \rightarrow \mathbf{Q} \otimes \mathbf{A}: a \mapsto 1 \otimes a
$$

is $\mathbf{1 - 1}$. Thus, $\mathbf{A}$ is just a $\mathbf{Z}$-submodule of $\mathbf{Q} \otimes \mathbf{A}$ considered as a $\mathbf{Z}$-module. Indeed, $\mathscr{K}$ is precisely the class of all $\mathbf{Z}$-submodules of $\mathbf{Q}$-modules (where the $\mathrm{Z}$-action is determined by restriction of scalars). Let $\mathscr{V}$ be the variety of all Q-modules. It can be shown that the restriction map is an isomorphism from $\mathbf{C o n}_{\mathscr{V}} \mathbf{Q} \otimes \mathbf{A}$ to $\mathbf{C o n}_{\mathscr{H}} \mathbf{A}$ for any $\mathbf{A} \in \mathscr{K}$. As $\mathscr{V}$ is congruence-modular, $\mathscr{K}$ is relatively modular.

In the example of the previous paragraph we identified $\mathbf{A}$ with a certain natural subreduct of $\mathbf{Q} \otimes \mathbf{A}$. By extending scalars from $\mathbf{Z}$ to $\mathbf{Q}$ we essentially brought in extra operations which were compatible with the $\mathscr{K}$-congruences of A but which destroyed all other congruences. This process also introduced new elements. We could not extend scalars too far or else we would not have been able to show that the restriction map from $\operatorname{Con}_{\mathscr{V}} \mathbf{Q} \otimes \mathbf{A}$ to $\mathbf{C o n}_{\mathscr{K}} \mathbf{A}$ was an isomorphism; mainly because too many new elements would be introduced. Using abstract nonsense we can frame these ideas more generally. We will be 
able to show that certain quasivarieties are relatively modular by identifying them as special quasivarieties of subreducts of algebras from modular varieties.

In order to formalize the remarks above, let us suppose that we have an interpretation of a variety $\mathscr{W}$ into a congruence-modular variety $\mathscr{V}$. That is, we have a clone homomorphism $\phi:$ Clo $\mathscr{W} \rightarrow$ Clo $\mathscr{V}$. Considering $\mathscr{W}$ and $\mathscr{V}$ as categories in the obvious way, there is a forgetful functor $G: \mathscr{V} \rightarrow \mathscr{W}$ which takes any $\mathscr{V}$-algebra, A, to its reduct to the operations in the image of $\phi$. Via $\phi$, a $\mathscr{W}$-algebra structure on $G(\mathbf{A})$ is determined. Now $G$ has a left adjoint $F$. If $\mathbf{B} \in \mathscr{W}$, we say that $F(\mathbf{B})$ is the (universal) $\mathscr{V}$-envelope of B. A presentation of $F(\mathbf{B})$ is obtained by taking a $\mathscr{W}$-presentation of $\mathbf{B}$ and using $\phi$ to interpret it as a $\mathscr{V}$-presentation. If $\mathbf{B} \in \mathscr{W}, \mathbf{A} \in \mathscr{V}$, and there is a one-to-one $\mathscr{W}$-homomorphism $f: \mathbf{B} \rightarrow G(\mathbf{A})$, then we will call $\mathbf{A}$ a $\mathscr{V}$ extension of $\mathbf{B}$. There is a natural $\mathscr{W}$-homomorphism $\eta_{B}: \mathbf{B} \rightarrow G F(\mathbf{B})$. It is easy to see that $\mathbf{B}$ has a $\mathscr{V}$-extension iff $\eta_{B}$ is injective.

Let $\mathscr{K}$ be the class of algebras $\mathbf{B} \in \mathscr{W}$ for which $\eta_{B}$ is injective. It is easy to verify that $\mathscr{K}$ is closed under $\boldsymbol{I}, \boldsymbol{S}, \boldsymbol{P}$, and $\boldsymbol{P}_{u}$, although not necessarily under $\boldsymbol{H}$. We call $\mathscr{K}$ the derived quasivariety of the adjunction $\langle F \mid G\rangle$. $\mathscr{K}$ is precisely the class of members of $\mathscr{W}$ that have a $\mathscr{V}$-extension.

Both $F(\mathbf{B})$ and $G F(\mathbf{B})$ have the same underlying sets; and congruences on $F(\mathbf{B})$ are at the same time congruences on $G F(\mathbf{B})$. The homomorphism $\eta_{B}: \mathbf{B} \rightarrow G F(\mathbf{B})$ gives us a way of converting congruences on $F(\mathbf{B})$ into congruences on $\mathbf{B}$. In fact, the restriction map

$$
\eta_{B}^{*}: \operatorname{Con} F(\mathbf{B}) \rightarrow \operatorname{Con}_{\mathscr{H}} \mathbf{B}: \theta \mapsto \eta^{-1}(\theta)
$$

is a meet-semilattice homomorphism. It is not hard to show that $\eta_{B}^{*}$ is onto; this follows from the universality of $F(\mathbf{B})$. So if $\eta_{B}^{*}$ preserves joins, then the lattice $\operatorname{Con}_{\mathscr{K}} \mathbf{B}$ is a homomorphic image of the modular lattice $\operatorname{Con} F(\mathbf{B})$. This would imply that $\mathbf{C o n}_{\mathscr{K}} \mathbf{B}$ is modular. Unfortunately, $\eta^{*}$ usually does not preserve joins.

We will say that $\mathscr{K}$ has unique $\mathscr{V}$-extensions if for every $\mathbf{B} \in \mathscr{K}$, when $f: \mathbf{B} \rightarrow G(\mathbf{A})$ is one-to-one, then the natural map $\tilde{f}: F(\mathbf{B}) \rightarrow \mathbf{A}$ is one-to-one. Saying that $\mathscr{K}$ has unique $\mathscr{V}$-extensions is equivalent to saying that for every $\mathbf{B} \in \mathscr{K}$, the restriction map $\eta_{B}^{*}: \operatorname{Con} F(\mathbf{B}) \rightarrow \operatorname{Con}_{\mathscr{K}} \mathbf{B}$ is injective. When $\eta_{B}^{*}$ is injective, it does preserve joins since any one-to-one, onto, meet-preserving map between lattices preserves joins. Hence, if $\mathscr{K}$ is the derived quasivariety of an adjunction between $\mathscr{W}$ and the modular variety $\mathscr{V}$, and $\mathscr{K}$ has unique $\mathscr{V}$-extensions, then $\mathscr{K}$ is relatively modular.

In the example described at the beginning of this section the variety $\mathscr{W}$ is the variety of all $\mathbf{Z}$-modules, $\mathscr{V}$ is the variety of all $\mathbf{Q}$-modules, and the interpretation $\phi$ is the one determined by the ring embedding of $\mathbf{Z}$ into $\mathbf{Q}$. The derived quasivariety is just $\mathscr{K}$ : the quasivariety of all torsion-free $\mathbf{Z}$-modules. One verifies that $\mathscr{K}$ has unique Q-module extensions, so this example is a special case of the scenario just described.

The next theorem and its proof show that every relatively modular quasivariety of semigroups is contained in a relatively modular quasivariety derived as above from the standard interpretation of some proper variety of semigroups into the variety of groups. Corresponding to each variety $\mathscr{W}$ of semigroups other than the variety of all semigroups, there is a relatively modular quasivariety $\mathscr{K}$ consisting of the group-embeddable members of $\mathscr{W} . \mathscr{K}$ has unique group extensions. 
Theorem 7.1. Every relatively modular quasivariety of semigroups satisfies the cancellation laws:

$$
x y \approx x z \rightarrow y \approx z, \quad y x \approx z x \rightarrow y \approx z
$$

and a nontrivial equation. Conversely, any quasivariety axiomatized by the cancellation laws and a nontrivial equation is relatively modular.

Proof. Assume that $\mathscr{K}$ is a relatively modular quasivariety of semigroups. We begin by showing that $\mathscr{K}$ satisfies the cancellation laws. First, let $\mathbf{A}=\langle x\rangle$ be a 1-generated semigroup in $\mathscr{K}$. If $\mathbf{A}$ is infinite, then $\mathbf{A}$ is the free 1-generated semigroup and this semigroup satisfies the cancellation laws. Otherwise, $\mathbf{A}$ is finite and $x^{m}=x^{n}$ for some $m<n$; we may assume that $m$ is the smallest number for which such a relation holds. If $m>1$, then $\alpha=\mathrm{Cg}\left(x^{m-1}, x^{n-1}\right)$ is a minimal congruence on $\mathbf{A}$ and $\operatorname{typ}\left(0_{A}, \alpha\right)=\mathbf{1}$. This contradicts Lemma 4.4. Hence $m=1$. A is a cyclic group; so $\mathbf{A}$ satisfies the cancellation laws.

Now suppose that $\mathscr{K}$ fails the bi-implication $x y \approx x z \leftrightarrow y x \approx z x$. Then there is a $\mathbf{B} \in \mathscr{K}$ that has elements $a, b, c$, where, say, $a b=a c$ but $b a \neq$ $c a$. Let $\mathbf{S}$ be the subsemigroup of $\mathbf{B}$ that is generated by $e=b a$ and $f$ $=c a$. Notice that $e^{2}=e f$ and $f^{2}=f e$. If $e=e^{m}$ for some $m>1$, then $f^{2}=f e=f e^{m}=f^{m+1}$. By the result of the last paragraph, $f=f^{m}$. In this case, $\mathbf{S}$ is isomorphic to the direct product of a two-element left-zero semigroup $\mathbf{U}$ and an $m$-element cyclic group. By the factorization theorem (Theorem 6.3), $\mathbf{U} \in \mathscr{K}$. But $\mathbf{U}$ is a finite simple semigroup of type 1. This contradicts Lemma 4.4; thus this case cannot occur. Hence, there is no $m>1$ for which $e=e^{m}$ or $f=f^{m}$. If, in addition, there are no positive integers $m, n$ such that $e^{m}=f^{n}$, then $\mathbf{S} \cong \mathbf{U} \times \mathbf{V}$, the product of the two-element left-zero semigroup and the free semigroup $\mathbf{V}$ on one generator. This gives a contradiction, just as above. Hence there are positive $m, n$ satisfying $e^{m}=$ $f^{n}$. This means that $e^{m+1}=e e^{m}=e f^{n}=e^{n+1}$, so $e^{m-n+1}=e$. Hence, $m$ $=n$. Let $k$ be the least number $m$ for which $e^{m}=f^{m}$. To briefly summarize our progress, we have gathered enough information to show that, for some $k$, $\mathbf{S}$ is the semigroup presented by

$$
\left\langle e, f \mid e^{2}=e f, f^{2}=f e, e^{k}=f^{k}\right\rangle .
$$

One can check that $\mathrm{Cg}(e, f) \cap \mathrm{Cg}\left(e^{k}, e^{k+1}\right)=0$. Hence,

$$
\operatorname{Cg}(e, f)^{\prime} \cap \mathrm{Cg}\left(e^{k}, e^{k+1}\right)^{\prime}=0 .
$$

But

$$
\mathrm{Cg}^{\mathbf{S}}\left(e^{k}, e^{k+1}\right)^{\prime} \supseteq \mathrm{Cg}^{\langle e\rangle}\left(e^{k}, e^{k+1}\right)^{\prime}=1_{\langle e\rangle} .
$$

Similarly, since $e^{k}=f^{k}$ and $e^{k+1}=f^{k+1}$,

$$
\mathrm{Cg}^{\mathbf{S}}\left(e^{k}, e^{k+1}\right)^{\prime} \supseteq \mathrm{Cg}^{\langle f\rangle}\left(f^{k}, f^{k+1}\right)^{\prime}=1_{\langle f\rangle} .
$$

The pairs $\left(e, e^{k}\right)$ and $\left(f^{k}, f\right)$ belong to $\mathrm{Cg}^{\mathbf{S}}\left(e^{k}, e^{k+1}\right)^{\prime}$ and $e^{k}=f^{k}$; so

$$
(e, f) \in \mathrm{Cg}^{\mathbf{S}}(e, f)^{\prime} \cap \mathrm{Cg}^{\mathbf{S}}\left(e^{k}, e^{k+1}\right)^{\prime} .
$$

This is a contradiction.

We are to the point where we can conclude that

$$
\mathscr{K} \models \forall x, y, z(x y \approx x z \leftrightarrow y x \approx z x) .
$$


If $\mathscr{K}$ does not satisfy the cancellation laws, then there is a $\mathbf{C} \in \mathscr{K}$ generated by distinct elements $u, v$, and $w$ such that $u v=u w$. Let $I=\{t \in C: t v=$ $t w\}$. Because of the bi-implication satisfied by $\mathscr{K}$, that we proved above, $I$ is a two-sided ideal in $\mathbf{C}$ which contains $\langle u\rangle$. Let $\theta_{I}$ denote the congruence on C whose only nontrivial block is the set $I$.

We claim that $\theta_{I} \cap \operatorname{Cg}(v, w)=0_{C}$. To prove it, note that if $r \in I$ and $(x, y) \in \operatorname{Cg}(v, w)$ then $r x=r y$. Hence if $(r, s) \in \theta_{I} \cap \mathrm{Cg}(v, w)$, then

$$
r^{2}=r s=s^{2}=s r \text {. }
$$

Assume that these equations hold and $r \neq s$. On the basis of what we proved about 1-generated semigroups in $\mathscr{K}$, it is easy to see that $\langle r, s\rangle$ is an infinite semigroup consisting of $r, s, r^{2}, r^{3}, \ldots$ (all of these elements distinct). Let $\Sigma_{s}$ be the set satisfying the relative shifting lemma for $\mathscr{K}$. Choose $\langle p, q\rangle \in \Sigma_{s}$ so that $p^{\mathbf{C}}(r, s, s, s) \neq q^{\mathbf{C}}(r, s, s, s)$. Since $p^{\mathbf{C}}(r, r, r, r)=q^{\mathbf{C}}(r, r, r, r)$, each of the terms $p$ and $q$, expressed as a product of variables, must have the same length. Since $p^{\mathbf{C}}(r, s, s, s) \neq q^{\mathbf{C}}(r, s, s, s)$, this length must be 1 . Thus we have, say, $p^{\mathbf{C}}(r, s, s, s)=r$ and $q^{\mathbf{C}}(r, s, s, s)=s$ and each of $p^{\mathbf{C}}, q^{\mathbf{C}}$ depends on only one variable; but this leads immediately to a contradiction, in view of the equations satisfied by $p^{\mathbf{C}}$ and $q^{\mathrm{C}}$. Thus, indeed, $\theta_{I} \cap \mathrm{Cg}(v, w)=$ $0_{C}$, as claimed.

Extending to $\mathscr{K}$-congruences, we have $\theta_{I}^{\prime} \cap \mathrm{Cg}(v, w)^{\prime}=0_{\mathbf{C}}$. Let $\mathbf{D}=$ $\mathbf{C} / \theta_{I}^{\prime} . \mathbf{D}$ is generated by $\langle\bar{u}, \bar{v}, \bar{w}\rangle$ and $\bar{u}$ is an absorbing element for $\mathbf{D}$. Since $\bar{v} \neq \bar{w}$, at least one of $\bar{v}$ or $\bar{w}$ is different from $\bar{u}$, say $\bar{v} \neq \bar{u}$. Now there is a pair of terms $\langle p, q\rangle \in \Sigma_{s}$ such that

$$
p^{\mathbf{D}}(\bar{v}, \bar{u}, \bar{u}, \bar{u}) \neq q^{\mathbf{D}}(\bar{v}, \bar{u}, \bar{u}, \bar{u}) .
$$

One of these elements, say $p^{\mathbf{D}}(\bar{v}, \bar{u}, \bar{u}, \bar{u})$, is not equal to $\bar{u}$. Hence the term $p$, expressed as a product of variables, contains only its first variable, and $p^{\mathbf{D}}$ does not depend on its last three variables. This means that

$$
q^{\mathbf{D}}(\bar{v}, \bar{u}, \bar{u}, \bar{v})=p^{\mathbf{D}}(\bar{v}, \bar{u}, \bar{u}, \bar{v})=p^{\mathbf{D}}(\bar{v}, \bar{u}, \bar{u}, \bar{u}) \neq \bar{u} .
$$

So $q^{\mathbf{D}}$ does not depend on its middle two variables. This yields the contradiction that

$$
p^{\mathbf{D}}(\bar{v}, \bar{v}, \bar{u}, \bar{u})=p^{\mathbf{D}}(\bar{v}, \bar{u}, \bar{u}, \bar{u}) \neq q^{\mathbf{D}}(\bar{v}, \bar{u}, \bar{u}, \bar{u})=q^{\mathbf{D}}(\bar{v}, \bar{v}, \bar{u}, \bar{u}) .
$$

This concludes our proof that $\mathscr{K}$ satisfies the cancellation laws.

To show that $\mathscr{K}$ must satisfy a nontrivial semigroup equation is not hard now. The equations satisfied by the terms in $\Sigma_{s}$ cannot all be trivial, for example. To show this, let $\langle p, q\rangle \in \Sigma_{s}$ be a pair such that $p^{\mathbf{A}}(a, b, b, b) \neq$ $q^{\mathbf{A}}(a, b, b, b)$ in some $\mathbf{A} \in \mathscr{K}$ with $a, b \in \mathbf{A}$. Using the associative law and the cancellation laws, we may assume that the leftmost variable of $p$ is different from the leftmost variable of $q$. Now assume that both $p(x, x, y, y) \approx$ $q(x, x, y, y)$ and $p(x, y, y, x) \approx q(x, y, y, x)$ are trivial semigroup equations; that is, that these equations hold in every semigroup. Assume also that the leftmost variable of $p(x, y, z, u)$ is $x$. Then the leftmost variable of $p(x, x, y, y)=q(x, x, y, y)$ is also $x$. Thus the leftmost variable of $q(x, y, z, u)$ is either $x$ or $y$. Applying the same reasoning to the equation $p(x, y, y, x) \approx q(x, y, y, x)$ shows that the leftmost variable of $q(x, y, z, u)$ is not $y$. Since $p$ and $q$ have different leftmost variables, we 
have a contradiction to the assumption that the leftmost variable of $p$ is $x$. Similar arguments rule out the possibility that the leftmost variable of $p$ is $y, z$, or $u$. This is a contradiction to the assumption that $\mathscr{K}$ satisfies no nontrivial equation. It proves the first statement of the theorem.

Now we prove that if $\mathscr{K}$ is axiomatized by the cancellation laws and one nontrivial semigroup equation, then $\mathscr{K}$ is relatively modular. Because of the cancellation laws, we may assume that the nontrivial equation is of the form

$$
x \rho_{0}(x, y, \bar{z}) \approx y \rho_{1}(x, y, \bar{z})
$$

for certain semigroup terms $\rho_{0}(x, y, \bar{z})$ and $\rho_{1}(x, y, \bar{z})$. Now, if $\mathbf{A} \in \mathscr{K}$ and $b, c \in \mathbf{A}$, then there exists $u, v \in \mathbf{A}$ such that $b u=c v$; we say that $\mathbf{A}$ is directed by left divisibility. In fact, we may choose $u=\rho_{0}(b, c, c, \ldots, c)$ and $v=\rho_{1}(b, c, c, \ldots, c)$. It is known, and this is Proposition 3.5 of [C], that any cancellation semigroup which is directed by left divisibility is embeddable in a group in which every element is expressible as a fraction, $a b^{-1}$, where $a, b \in$ $\mathbf{A}$, and that this group of fractions is uniquely determined by $\mathbf{A}$. Further, it is clear that if $\mathbf{A}$ is a subsemigroup of any group $\mathbf{G}$, then the set of fractions of elements of $A$ forms a subgroup of $\mathbf{G}$. (To show that this set of fractions is closed under multiplication notice that

$$
\left(a b^{-1}\right)\left(c d^{-1}\right)=a\left(b^{-1} c\right) d^{-1}=a\left(u v^{-1}\right) d^{-1}=(a u)(d v)^{-1},
$$

where $b u=c v$.) Hence, if $\mathbf{A}$ is a subsemigroup of any group $\mathbf{H}$ which is generated by $A$ (as a group), then $\mathbf{H}$ is the unique group of fractions of $\mathbf{A}$. In particular, such an $\mathbf{H}$ can only be the universal group of $\mathbf{A}$. The restriction map of $\theta \in \operatorname{Con} \mathbf{H}$ to $\left.\theta\right|_{A} \in \operatorname{Con} \mathbf{A}$ is one-to-one, since $\theta$ is determined by the set of pairs $\left(a b^{-1}, 1\right) \in \theta$, and $\left.\left(a b^{-1}, 1\right) \in \theta \leftrightarrow(a, b) \in \theta\right|_{A}$. It follows that $\mathscr{K}$ has unique group extensions, in the technical sense defined before this theorem. We have that $\operatorname{Con}_{\mathscr{K}} \mathbf{A} \cong \mathbf{C o n} \mathbf{H}$ via restriction, and this lattice is modular. Since A was an arbitrary member of $\mathscr{K}$, this quasivariety is relatively modular.

Corollary 7.2. If $\mathscr{K}$ is a finitely generated, relatively modular quasivariety of semigroups, then $\mathscr{K}$ is finitely axiomatizable.

Proof. This is immediate from Theorem 7.1 and Corollary 3.2.

The approach that we used in Theorem 7.1 can be used to show that the quasivariety of additively cancellative semirings is relatively modular, by considering the obvious adjunction with the variety of rings. One can show that the quasivariety of additively cancellative semimodules over a given semiring is relatively modular by considering the obvious adjunction with a variety of modules. One can show that the quasivariety of (unital) rings of characteristic 0 is relatively modular by considering the obvious adjunction with the variety of $\mathbf{Q}$-algebras, where $\mathbf{Q}$ denotes the rational numbers. Some readers may be able to conjure up a long list of their own examples of relatively modular quasivarieties in this way.

In our discussion of the adjunction between $\mathscr{W}$ and $\mathscr{V}$ before Theorem 7.1 , we made the unnecessary assumption that $\mathscr{W}$ and $\mathscr{V}$ are varieties. It is only necessary that they be quasivarieties. For example, if $\mathscr{V}$ is the quasivariety of torsion-free Abelian groups and $\mathscr{W}$ is the quasivariety of cancellation semigroups, and if we use the usual adjunction between semigroups and groups, then we obtain that the quasivariety of commutative, torsion-free semigroups with cancellation is relatively modular. 
Another unnecessary assumption that we made is that $\mathscr{V}$ is congruencemodular. This technique of producing examples by adjunction shows that any sentence expressible in the language of lattice theory which holds in the congruence lattices of algebras in $\mathscr{V}$ holds as a relative congruence condition for the algebras in the derived quasivariety $\mathscr{K}$, when the algebras in $\mathscr{K}$ have unique $\mathscr{V}$-extensions. This is because we end up showing that for each $\mathbf{B} \in \mathscr{K}$, $\operatorname{Con}_{\mathscr{K}} \mathbf{B} \cong \operatorname{Con} F(\mathbf{B})$, where $F(\mathbf{B}) \in \mathscr{V}$.

\section{BIBLIOGRAPHY}

[C] P. M. Cohn, Universal algebra, Reidel, 1965.

[CzDz] J. Czelakowski and W. Dziobiak, Congruence distributive quasivarieties whose finitely subdirectly irreducible members form a universal class, Algebra Universalis 27 (1990), 128-149.

[D] B. Davey, Monotone clones and congruence-modularity, preprint.

[DQS] B. Davey, R. W. Quackenbush, and D. Schweigert, Monotone clones and the varieties they determine, preprint.

[Dz1] W. Dziobiak, On infinite subdirectly irreducible algebras in locally finite equational classes, Algebra Universalis 13 (1981), 393-394.

[Dz2] _ Finitely generated congruence-distributive quasivarieties of algebras, Fund. Math. 133 (1989), 47-57.

[Dz3] Relative congruence-distributivity within quasivarieties of nearly associative $\phi$-algebras, Fund. Math. 135 (1990), 77-95.

[FM] R. Freese and R. McKenzie, Commutator theory for congruence modular varieties, London Math. Soc. Lecture Note Ser., No. 125, Cambridge Univ. Press, 1987.

[GL] G. Grätzer and H. Lakser, The lattice of quasivarieties of lattices, Algebra Universalis 9 (1979), 102-115.

[HM] D. Hobby and R. McKenzie, The structure of finite algebras, Contemp. Math., Amer. Math. Soc., Providence, R.I., 1988.

[K1] K. Kearnes, Natural examples of quasivarieties with EDPM, preprint.

[K2] Relatively congruence distributive subquasivarieties of a congruence modular variety, Bull. Austral. Math. Soc. 41 (1990), 87-96.

[M] R. McKenzie, Finite equational bases for congruence modular varieties, Algebra Universalis 24 (1987), 224-250.

[O] A. Yu. Ol'shanskii, Conditional identities in finite groups, Sibirsk. Mat. Zh. 15 (1974), 1409-1413, 1432; English transl. in Siberian Math. J. (1975).

[P] D. Pigozzi, Finite basis theorems for relatively congruence-distributive quasivarieties, Trans. Amer. Math. Soc. 310 (1988), 499-533.

Department of Mathematics, Vanderbilt University, Nashville, Tennessee 37240

Department of Mathematics, University of California, Berkeley, California 94720 